\title{
Recognition of Late Triassic Cu-Mo Mineralization in the Northern Yidun Arc (S.E. Tibetan Plateau): Implications for Regional Exploration
}

\author{
Zhen-Dong Tian ${ }^{1,2}$, Cheng-Biao Leng ${ }^{1,3, * \mathbb{D}}$, Xing-Chun Zhang ${ }^{1}$, Li-Min Zhou ${ }^{4}$ \\ and Yan-Wen Tang ${ }^{1}$ \\ 1 State Key Laboratory of Ore Deposit Geochemistry, Institute of Geochemistry, Chinese Academy of Sciences, \\ Guiyang 550081, China; tianzhendong@mail.gyig.ac.cn (Z.-D.T.); zhangxingchun@vip.gyig.ac.cn (X.-C.Z.); \\ tangyanwen@vip.gyig.ac.cn (Y.-W.T.) \\ 2 College of Earth and Planetary Sciences, University of Chinese Academy of Sciences, Beijing 100049, China \\ 3 State Key Laboratory of Nuclear Resources and Environment, East China University of Technology, \\ Nanchang 330013, China \\ 4 National Research Center for Geoanalysis, Chinese Academy of Geological Sciences, Beijing 100037, China; \\ limin.zhou@cags.ac.cn \\ * Correspondence: lengchengbiao@vip.gyig.ac.cn or lcb8207@163.com; Tel.: +86-1898-417-7223
}

Received: 29 October 2019; Accepted: 5 December 2019; Published: 10 December 2019

\begin{abstract}
The Yidun arc, located in the southeastern Tibetan Plateau, was formed by the westward subduction of the Ganze-Litang Paleo-Tethys ocean in Late Triassic. It is well-known for the formation of numerous Mesozoic porphyry-skarn $\mathrm{Cu}-\mathrm{Mo}-(\mathrm{Au})$ deposits in the arc. To date, more than 20 $\mathrm{Cu}-\mathrm{Mo}-(\mathrm{Au})$ deposits ( $>10$ million tonnes $\mathrm{Cu}$ resources) have been discovered in the southern Eastern Yidun arc. However, few $\mathrm{Cu}-\mathrm{Mo}$ deposits have been discovered in the northern Eastern Yidun arc. In recent years, some $\mathrm{Cu}-\mathrm{Mo}$ deposits or occurrence are successively discovered in the northern Eastern Yidun arc, but their ore-forming ages are not well constrained. It remains unclear whether such $\mathrm{Cu}-\mathrm{Mo}$ mineralization formed by similar metallogenic event and geodynamic setting as the $\mathrm{Cu}-\mathrm{Mo}-(\mathrm{Au})$ mineralization in the south. In order to determine the metallogenic age and shed light on potential links between $\mathrm{Cu}$-Mo mineralization and regional magmatic events, we present molybdenite Re-Os and zircon $\mathrm{U}-\mathrm{Pb}$ ages to constrain the timing of two types of $\mathrm{Cu}-\mathrm{Mo}$ mineralization in the northern Eastern Yidun arc (type I and type II). Molybdenite ICP-MS Re-Os dating results show that type I mineralization was formed at $217.7 \pm 3.6 \mathrm{Ma}$, which is highly consistent with the formation ages of the host granite $(218.1 \pm 1.5 \mathrm{Ma}, 2 \sigma, \mathrm{n}=15, \mathrm{MSWD}=0.92)$ and aplite dyke $(217.3 \pm 1.3 \mathrm{Ma}$, $2 \sigma, \mathrm{n}=16, \mathrm{MSWD}=0.50$ ) within error. While the type II mineralization has a relatively younger formation age of $211.8 \pm 4.7 \mathrm{Ma}$ than the host granite $(217.1 \pm 1.5 \mathrm{Ma}, 2 \sigma, \mathrm{n}=14, \mathrm{MSWD}=0.96)$ and type I Cu-Mo mineralization. These data indicate that the $\mathrm{Cu}-\mathrm{Mo}$ mineralization in the northern Eastern Yidun arc was temporally and spatially related to the Late Triassic magmatism in the region. Rhenium (Re) concentrations in the molybdenite from type I mineralization, ranging from 12.77 to $111.1 \mathrm{ppm}$ (typically $>100 \mathrm{ppm}$ ), indicate that the ore-forming metals were derived mainly from a mantle source. However, Re contents in molybdenite from the type II mineralization, ranging from 7.983 to $10.40 \mathrm{ppm}$, indicate that the ore-forming metals were derived from a mixed mantle and crustal source with a predominantly crustal component. This study confirms that the northern Eastern Yidun arc exists Late Triassic $\mathrm{Cu}$-Mo metallogenesis, and thus much attention should be paid on this region to find more Late Triassic Cu-Mo resources.
\end{abstract}

Keywords: Yidun arc; Triassic Cu-Mo mineralization; zircon U-Pb dating; molybdenite Re-Os dating; exploration 


\section{Introduction}

The Yidun arc is one of the largest volcanic island arcs in the Sanjiang Tethyan Metallogenic Domain of the southeastern Tibetan Plateau (Figure 1a) [1,2]. The arc was developed through two phases of magmatic activity in Late Triassic and Late Cretaceous, respectively (Figure 1a), which contributed to the formation of numerous deposits and occurrences [2-4]. In the region, more than 20 porphyry, skarn, and quartz-vein type $\mathrm{Cu}-\mathrm{Mo}-(\mathrm{Au}-\mathrm{W})$ polymetallic deposits of various sizes have been discovered and explored (Figure 1b) [5-11]. The porphyry Cu-Mo-Au deposits include the giant Pulang Cu-Au deposit, the large Xuejiping $\mathrm{Cu}$-Au deposit, and several medium to small $\mathrm{Cu}$ deposits (e.g., Lannitang, $\mathrm{Chundu}$, Songnuo) (Figure 1b) $[6,12,13]$. The skarn Cu-Mo deposits include two large Cu-Mo deposits (Hongshan and Tongchanggou), and several medium to small Cu-Mo deposits (e.g., Langdu, Gaochiping) $[8,9]$. The quartz-vein type W-Mo deposits include the Relin and Xiuwacu W-Mo deposits with medium scale (Figure 1b) $[5,14,15]$. However, these deposits are exclusively clustered in the Southern Eastern Yidun Arc (SEYA). Recently, some porphyry Cu-(Mo-Au) deposits (e.g., Changdagou, Zhujiding; Figure 1a) have been successively discovered in the Northern Eastern Yidun Arc (NEYA) [16-18], indicating that the NEYA may also have potential to find Cu-Mo resources. However, the ore-forming ages of the $\mathrm{Cu}$-Mo deposits or occurrences have not been well constrained, which hampers our understanding of the relationship between the $\mathrm{Cu}-\mathrm{Mo}$ mineralization and regional magmatism. In addition, the lack of ore-forming age also obscures our prospecting target where should we put effort into finding Cu-Mo deposit, in late Triassic intrusions or in late Cretaceous intrusions?

Molybdenite Re-Os and zircon U-Pb isotopic systems have high closure temperature and have proven to be powerful tools to determine the precise metallogenic age [5,19-21]. In this study, we present new molybdenite Re-Os and zircon LA-ICP-MS U-Pb ages to constrain the timing of Cu-Mo mineralization and their host granitic rocks, respectively. This data provides first constraints on the timing of $\mathrm{Cu}$-Mo mineralization, the source of ore-forming metals, as well as the implications for the regional exploration.

\section{Regional Geology}

The Yidun arc, situated in the southeastern margin of the Tibetan Plateau (Figure 1a). To the west, it is bounded by the Jinshajiang suture which is considered to be a Late Paleozoic Paleo-Tethyan oceanic subduction zone dipping to the west (Figure 1a) [22]. To the east, it is bounded by the Ganze-Litang suture which is considered to be a westward-dipping Paleo-Tethyan oceanic subduction zone during the Middle-Late Triassic (Figure 1a) [22]. The Yidun arc was formed by the westward subduction of the Ganze-Litang Paleo-Tethys ocean in Late Triassic [22-24]. Tectonically, the arc can be divided into two principal geological units, the Western Yidun arc (WYA) and Eastern Yidun arc (EYA), by the NNW-trending Xiangcheng-Geza fault (Figure 1a). The WYA, also named as Zhongza massif, consists of Paleozoic shallow to deep marine carbonates and clastic rocks interlayered with volcanic rocks, comparable to the Paleozoic passive continental margin sedimentary sequences of the western Yangtze Block. Based on the similarity of Paleozoic successions and paleontological fossils between the Yidun arc and Yangtze Block, it was traditionally considered that the WYA was rifted from the Yangtze Block during Middle to Late Paleozoic due to the opening of the Ganze-Litang Paleo-Tethyan ocean $[25,26]$. During Middle to Late Triassic, the Paleozoic sedimentary rocks in the WYA have undergone collision-related greenschist to lower amphibolite facies metamorphism due to the closure of the Jinshajiang Paleo-Tethys ocean, which subsequently led to the collision of WYA with the Qiangtang terrane [1,22]. The EYA consists of sporadically exposed Precambrian metamorphic basement, and Paleozoic to Triassic sedimentary covers [23]. The basement rocks include schist, leptynite, quartzite, marble, and felsic volcanic rocks interlayers $[27,28]$. The Paleozoic strata are composed of clastic rocks, shallow to deep marine carbonates intercalated with mafic volcanic rocks [23].The Late Triassic strata, from the base upward, include the Qugasi Formation, Tumugou Formation, Lanashan Formation, and Lamaya Formation [23]. The Qugasi Formation is composed of sandstone, slate, phyllite, limestone and mafic arc volcanic rocks [23,29]. These rocks are intruded 
by $\sim 230 \mathrm{Ma}$ (zircon U-Pb age) quartz diorite, indicating that the deposition time of the Qugasi Formation should be older than 230 Ma [29]. The Tumugou Formation, conformably overlying the Qugasi Formation, comprises conglomerate sandstone, slate, limestone, intermediate to felsic arc volcanic rocks (e.g., rhyolite, and andesite) $[1,23,29,30]$. LA-ICP-MS zircon U-Pb dating show that the volcanic rocks in the lower and upper Tumugou Formation were erupted at $\sim 230 \mathrm{Ma}$ and $220 \mathrm{Ma}$, respectively [1,30], indicating that the Tumugou Formation was deposited at 230-220 Ma.
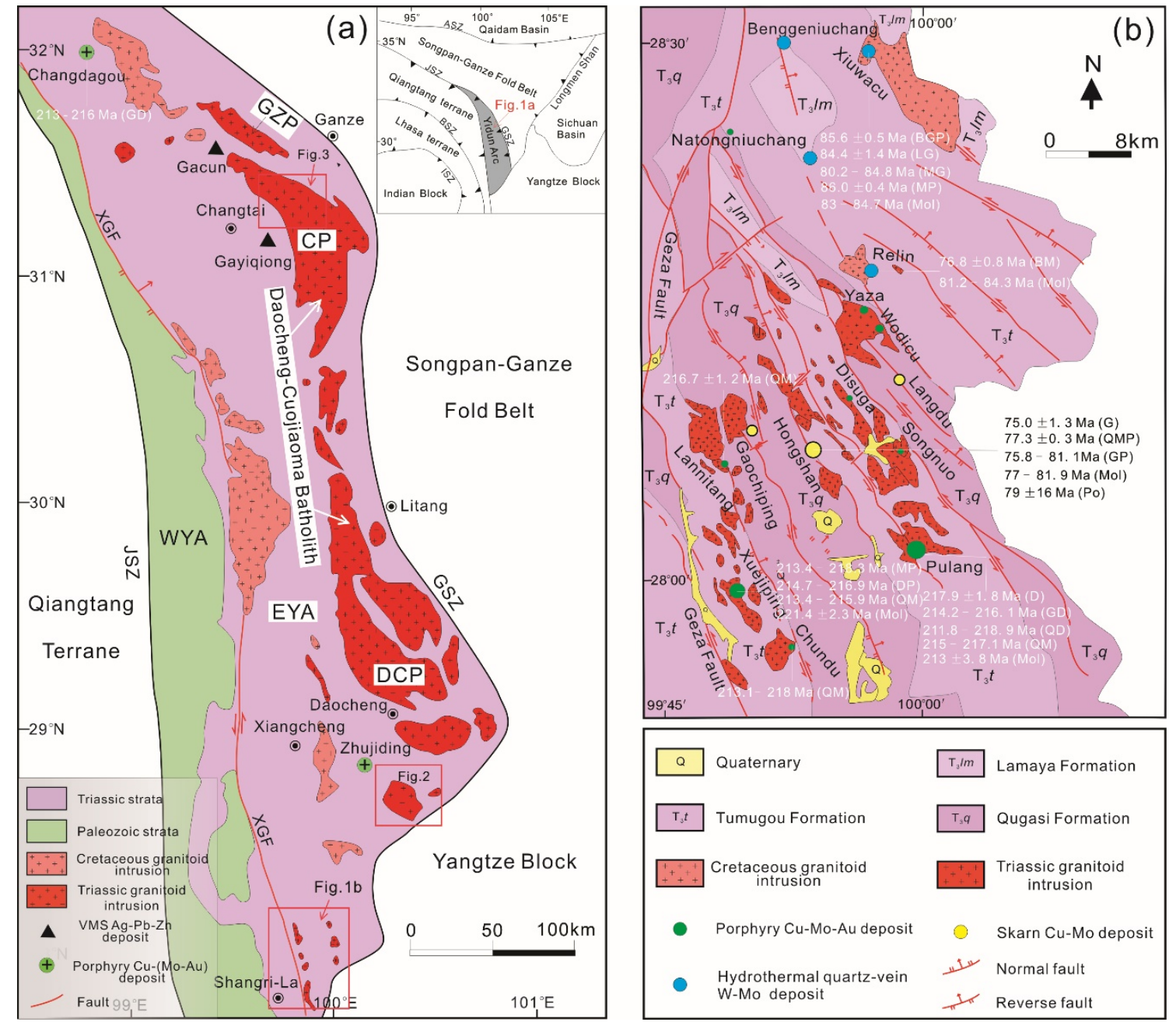

Figure 1. (a) Simplified geologic map of the Yidun arc (modified after Wang et al. [30]). (b) Geologic map of the SEYA (modified after Leng et al. [6]). Abbreviations: ASZ = Animaqin suture zone, $\mathrm{BSZ}=$ Bangong-Nujiang suture zone, GSZ = Ganze-Litang suture zone, ISZ = Indus-Yarlung suture zone, JSZ = Jinshajiang suture zone, CP = Cuojiaoma pluton, DCP = Daocheng pluton, GZP = Ganze pluton, EYA = Eastern Yidun arc, WYA = Western Yidun arc, XGF = Xiangcheng-Geza fault. $\mathrm{BGP}=$ Biotite granitic porphyry, $\mathrm{BM}=$ Biotite monzogranite, $\mathrm{D}=$ Diorite, $\mathrm{DP}=$ Diorite porphyry, $\mathrm{G}=$ Granite, GD = Granodiorite, GP = Granite porphyry, LG = Leucogranite, MG = Monzogranite, $\mathrm{Mol}=$ Molybdenite, $\mathrm{MP}=$ Monzonite porphyry, $\mathrm{Po}=$ Pyrrhotite, $\mathrm{QD}=$ Quartz diorite, $\mathrm{QM}=$ Quartz monzonite, QMP = Quartz monzonite porphyry. The age data are from Table 4.

The Lanashan Formation is in conformable contact with the Tumugou Formation and consists of sandstone, slate, limestone, mafic volcanic rocks, and conglomerate in the bottom $[23,29]$. Conformably overlying the Lanashan Formation, the Lamaya Formation is dominant by the dark slate and sandstone [23,29]. The arc volcanic rocks interlayered in the Late Triassic strata have been interpreted as the products of the westward subduction of the Ganze-Litang Paleo-Tethyan ocean $[1,30]$. The Late Triassic volcanic-sedimentary successions were intruded by voluminous intermediate to felsic intrusions 
(225-215 Ma; see below). In addition to Tertiary and Quaternary sediments, other strata younger than Triassic (e.g., Jurassic and Cretaceous) are absent in the whole Yidun arc, though there is belt of Cretaceous granites in the EYA (88-80 Ma; see below). The EYA was collided with the Songpan-Ganze Fold Belt at the end of the Triassic owing to the closure of the Ganze-Litang Paleo-Tethyan ocean [29]. Following the collision of India with Asia during the Tertiary, the Yidun arc was incorporated into the modern Tibetan Plateau [6].

As stated above, magmatism in the EYA was principally emplaced during the Late Triassic and Late Cretaceous times [4,10]. The Late Triassic intrusions intruded into the Upper Triassic volcanic-sedimentary successions in the northern part of Yidun arc. They are composed of biotite monzogranite, granodiorite and quartz diorite. Previous studies show that the intrusions emplaced at ca. 225-215 Ma (zircon U-Pb age) [3,31,32]. These granitic rocks are metaluminous or slightly peraluminous and belong to high-K calc-alkaline I-type granitoid [3], with negative to positive $\varepsilon_{\mathrm{Hf}(\mathrm{t})}$ values ( -9.8 to 3.4$)$ and negative $\varepsilon_{\mathrm{Nd}(\mathrm{t})}$ values ( -7.8 to -5.7$)$ [3]. They have been interpreted to be partial melting products of Late Paleoproterozoic to Early Mesoproterozoic mafic-intermediate lower crust with minor involvement of mantle-derived materials [3]. In the southern segment of the EYA, the Late Triassic magmatic rocks are composed of granodiorite, monzonite, quartz diorite porphyry, quartz monzonite porphyry, andesite, formed at 221-211 Ma (zircon U-Pb ages) [33]. These intermediate-felsic plutonic rocks host numerous porphyry Cu-Mo-Au deposits (e.g., Pulang, Xuejiping, Chundu, Disuga), and skarn Cu-Mo deposits (e.g., Langdu; Figure 1b). The detailed relationship between the Late Triassic magmatism and regional $\mathrm{Cu}-\mathrm{Mo}-\mathrm{Au}$ mineralization has been reviewed by Li et al. [4].

The Late Cretaceous intrusions in the EYA were formed at 88-80 Ma (zircon U-Pb ages) along an N-S trending fault (Figure 1a). They consist of granite, monzogranite, biotite granite, and granitic porphyry [4]. These igneous rocks are characterized with high $\mathrm{SiO}_{2}$ and $\mathrm{K}_{2} \mathrm{O}+\mathrm{Na}_{2} \mathrm{O}$ contents, enrichment in large ion lithophile element (LILE) and depletion in high field strength element (HFSE), with variable negative $\varepsilon_{\mathrm{Hf}(\mathrm{t})}(-7.6$ to -3.2$)$ and $\varepsilon_{\mathrm{Nd}(\mathrm{t})}(-8.0$ to -6.9$)$ values. These intrusions host a series of skarn Cu-Mo deposits (e.g., Hongshan) and quartz vein Mo-W deposits (e.g., Relin and Xiuwacu) in the south segment of the EYA (Figure 1b) $[4,5,7,9,11,14,34]$. The detailed relationship between the Late Cretaceous magmatism and regional $\mathrm{Cu}-\mathrm{Mo}-\mathrm{W}$ mineralization has been reviewed by $\mathrm{Li}$ et al. [4].

The distribution of the Late Triassic and Late Cretaceous magmatic rocks and their associated deposits are controlled by the Late Triassic NW-trending and NE-trending faults [4]. These NWand NE-trending faults are regarded as the main channel of ore-forming fluid migration [4]. The NW-trending faults are reverse fault, with strike direction of $\sim 320-350^{\circ}$ and the dip direction of NE [23]. The NW-trending faults were cut the NE-trending faults (Figures $1 b, 2$ and 3 ). The NE-trending faults belong to normal faults. They have strike directions of $65-85^{\circ}$ or $30-50^{\circ}$, with the dip direction of SE [23]. 


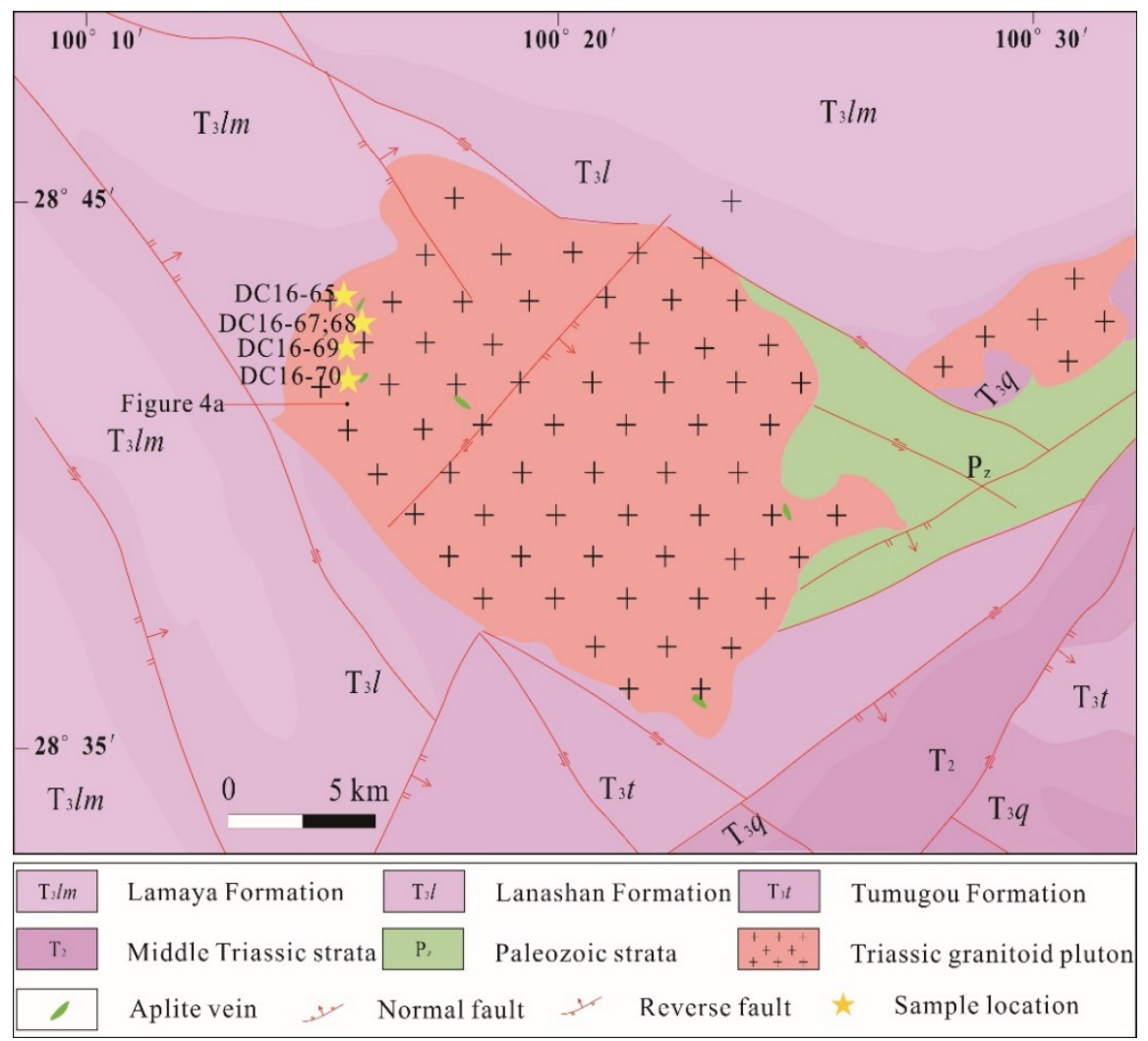

Figure 2. Simplified geological map of type I Cu-Mo mineralization in the NEYA (modified from Chen et al. [35]).

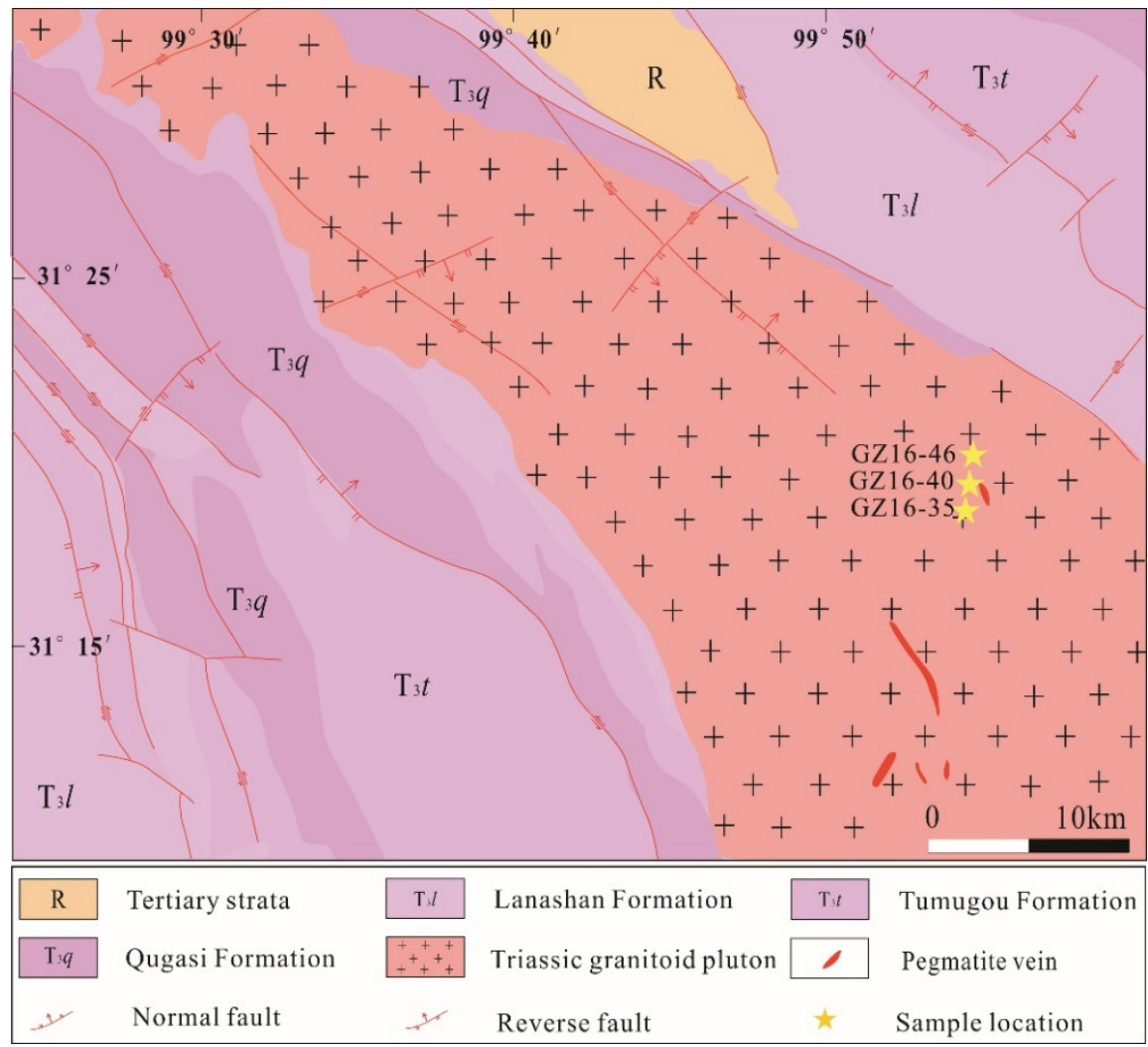

Figure 3. Simplified geological map of type II Cu-Mo mineralization in the NEYA (modified from Gao et al. [36]). 


\section{Sampling and Analytical Methods}

\subsection{Sampling and Sample Descriptions}

Field and hand sample observations show that the type I Cu-Mo mineralization $\left(100^{\circ} 15^{\prime} 31.7^{\prime \prime} \mathrm{E}\right.$, $28^{\circ} 43^{\prime} 33.8^{\prime \prime} \mathrm{N}$; Figure 2) is located within the contact surface between the granite and the granitic aplite (Figure $4 \mathrm{a}, \mathrm{c}-\mathrm{e}$ ). The granite intrudes the strata of the Lamaya and Lanashan formations and is itself cut by granitic aplite dykes (Figures 2 and $4 \mathrm{a}$ ). Molybdenite is the major sulfide in the type I $\mathrm{Cu}-\mathrm{Mo}$ mineralization and occurs as aggregates or thin coating of the contact surfaces (Figure $4 \mathrm{~d}, \mathrm{e}, \mathrm{i}$ ). Chalcopyrite and pyrite are also observed in the type I Cu-Mo mineralization (Figure 4i). The type II Cu-Mo mineralization $\left(99^{\circ} 54^{\prime} 25.5^{\prime \prime} \mathrm{E}, 31^{\circ} 19^{\prime} 19.3^{\prime \prime} \mathrm{N}\right.$; Figure 3$)$ is distributed within the veins and cracks in granite (Figure $4 \mathrm{f}-\mathrm{h}$ ). The granite intrudes the strata of Lanashan and Qugasi formations (Figure 3).
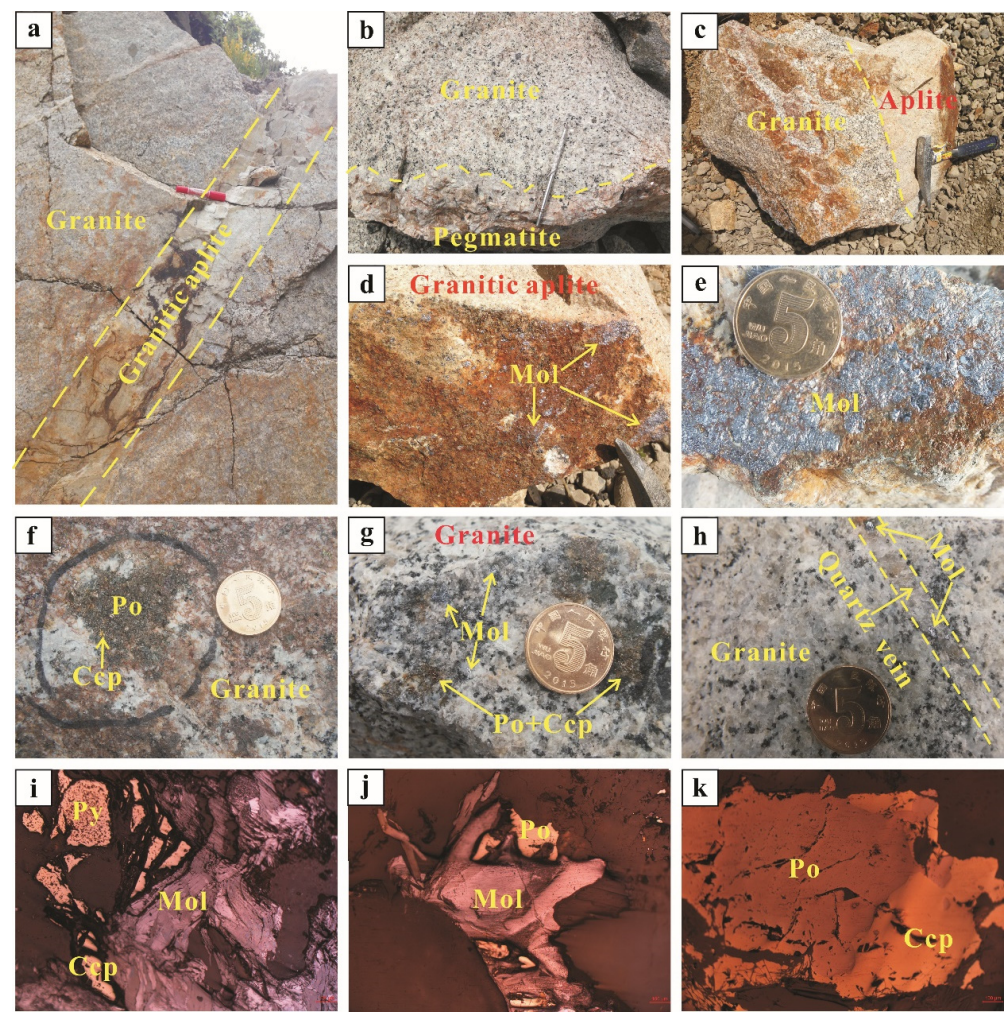

Figure 4. Representative photographs of host granites, granitic aplite, and two types of $\mathrm{Cu}-\mathrm{Mo}$ mineralization in the NEYA. (a) Representative field outcrop of the host granite and granitic aplite of type I Cu-Mo mineralization. (b) Typical host rock of type II Cu-Mo mineralization and the granite is spatially associated with granitic pegmatite. (c) Typical hand specimen of granite that was crosscut by granitic aplite. Sulfides are distributed along the crack surface between aplite and granite. (d,e) Thin coating of molybdenite within the contact surface between the granite and granitic aplite of type I Cu-Mo mineralization. (f,g) Molybdenite, chalcopyrite, and pyrrhotite in fracture surface of granite. (h) Molybdenite-bearing quartz vein in granite of type II Cu-Mo mineralization. (i) Molybdenite coexists with chalcopyrite and pyrite of type I Cu-Mo mineralization. ( $\mathbf{j}, \mathbf{k})$ Molybdenite coexists with chalcopyrite and pyrrhotite of type II Cu-Mo mineralization. Abbreviations: Ccp-chalcopyrite, Mol—molybdenite, Po-pyrrhotite, Py-pyrite.

The main ore mineral assemblages are molybdenite, pyrrhotite, and chalcopyrite. Molybdenite is disseminated or occurs as speckles within the fractures of the host granite (Figure $4 \mathrm{j}, \mathrm{k}$ ). In this study, two granites (DC16-68 and GZ16-40), one granitic aplite (DC16-65), and seven molybdenite samples (DC16-67, DC16-68, DC16-69, DC16-70, GZ16-35, GZ16-40, and GZ16-46) were collected from two types of $\mathrm{Cu}-\mathrm{Mo}$ occurrences for zircon LA-ICP-MS U-Pb dating and ICP-MS Re-Os isotopic analyses 
(Figures 2 and 3). The granites are gray and exhibit a granitic structure and massive texture. They are composed of euhedral plagioclase (50-55 vol.\%), anhedral quartz (35-40 vol.\%), and small amounts of euhedral biotite and hornblende (10-15 vol.\%).

Accessory minerals include apatite, titanite, and zircon. The aplitic dyke, crosscutting the host granite of type I Cu-Mo mineralization, is light-gray, 5-15 cm wide (Figure 4a,c), and exhibits fine-grained structure and massive texture. It has a sharp contact with the granite (Figure $4 a, c)$.

\subsection{LA-ICP-MS U-Pb Dating}

Zircon grains for Laser Ablation Inductively Coupled Plasma Mass Spectrometry (LA-ICP-MS) $\mathrm{U}-\mathrm{Pb}$ dating were separated using conventional magnetic and heavy liquid techniques and then handpicked under a binocular microscope. They were then mounted in epoxy resin on a $2 \mathrm{~cm}$ diameter disk, which would be polished to section the crystals in half for analyses. Prior to in-situ U-Pb isotopic analyses, all zircons were examined under transmitted and reflected light with an optical microscope at the State key Laboratory of Ore Deposit Geochemistry (SKLODG), Institute of Geochemistry, Chinese Academy of Sciences. Cathodoluminescence (CL) images were obtained using a JSM-7088F type thermal field scanning electron microscope equipped with a Gatan Mono CL4 detector at the SKLODG. Based on the transmitted and reflected light, and BSE observations, the inclusion-free domains were selected for the U-Pb isotopic analyses.

A 7900 ICP-MS (Agilent, Santa Clara, CA, USA) equipped with a GeoLas Pro $193 \mathrm{~nm}$ ArF excimer laser at SKLODG was used to measure the U-Pb ages of zircon. Helium was used as carrier gas mixed with argon via a T-connector before entering the spectrometer. A $32 \mu \mathrm{m}$ laser spot size was selected during the ablation with a repetition rate of $5 \mathrm{~Hz}$. Each analysis consists of $20 \mathrm{~s}$ background signal acquisition followed by $50 \mathrm{~s}$ ablation signal acquisition. Zircon 91500 was used as external standard to correct elemental fractionation and zircon GJ-1 and Plešovice were analyzed as quality controls. NIST SRM 610 glass was used as external standard to normalize $\mathrm{U}, \mathrm{Th}, \mathrm{Pb}$ contents, with zircon ${ }^{29} \mathrm{Si}$ concentrations used for internal standardization. The LA-ICP-MS zircon U-Pb dating results of standard zircons are listed in Table 1. Standard zircon 91500, GJ-1 and Plešovice yielded concordia age of $1062.6 \pm 2.7 \mathrm{Ma}(\mathrm{MSWD}=0.08, \mathrm{n}=22), 599.7 \pm 2.3 \mathrm{Ma}(\mathrm{MSWD}=0.07, \mathrm{n}=7)$, and $339.2 \pm 4.4 \mathrm{Ma}$ $(\mathrm{MSWD}=2.4, \mathrm{n}=7)$, respectively.

These ages are coinciding with the recommended ${ }^{206} \mathrm{~Pb} /{ }^{238} \mathrm{U}$ ages of standard zircon 91500 (1062.4 \pm 0.8 Ma [37]), GJ-1 (599.8 $\pm 4.5 \mathrm{Ma}$ [38]), and Plešovice (337.13 $\pm 0.37 \mathrm{Ma}$ [39]) within error. Off-line raw data selection and integration of background and analytic signals, time-drift correction and quantitative calibration for U-Pb dating were performed by ICPMSDataCal program $[40,41]$. The age calculations, the plotting of concordia diagrams (Figure 6a,c,e) and weighted mean age diagrams (Figure 6b,d,f) were made using Isoplot Ver_3.0 [42]. The complete U-Pb dating results from three samples are listed in Table 2.

\subsection{ICP-MS Re-Os Dating}

Molybdenite samples were separated by hand-picking. Fresh and unoxidized molybdenite powders $(<0.1 \mathrm{~mm}$ in size and purity $>99 \%)$ were used for Re-Os isotopic analyses. The Re-Os isotopic analyses were performed at the Re-Os Laboratory of the National Research Center of Geoanalysis, Chinese Academy of Geological Science. Detailed operated processes, including sample preparation, chemical separation and mass spectroscopy, were done according to Du et al. [43] and Shirey and Walker [44]. Re and Os concentrations were determined by TJA X-series ICP-MS. The procedural blanks for this analysis were $0.0010( \pm 0.0011)$ for Re and 0.0001 for Os, which are far less than the contents of Re and Os in the analyzed molybdenite samples. The molybdenite standard sample GBW04435(HLP) yielded a model age of $220.5 \pm 3.0 \mathrm{Ma}$, which is in good agreement with the certified value $(221.4 \pm 5.6 \mathrm{Ma})$ within error. The molybdenite model age was calculated by the formula of $t=\left[\ln \left(1+{ }^{187} \mathrm{Os} /{ }^{187} \mathrm{Re}\right)\right] / \lambda$, where $\lambda$ is the ${ }^{187} \operatorname{Re}$ decay constant of $1.666 \times 10^{-11}$ per year [45]. The Re-Os isochron (Figure 7a,c) and weighted mean age (Figure 7b,d) were calculated and plotted by Isoplot Ver_3.0 [42]. 
Table 1. LA-ICP-MS zircon U-Pb dating results of standard zircons of 91500, GJ-1, and Plešovice.

\begin{tabular}{|c|c|c|c|c|c|c|c|c|c|c|c|c|c|c|c|c|}
\hline \multirow{2}{*}{ No. } & \multirow{2}{*}{ Th (ppm) } & \multirow{2}{*}{$\mathrm{U}(\mathrm{ppm})$} & \multirow{2}{*}{$\mathrm{Th} / \mathrm{U}$} & \multicolumn{2}{|c|}{${ }^{207} \mathrm{~Pb} /{ }^{206} \mathrm{~Pb}$} & \multicolumn{2}{|c|}{${ }^{207} \mathrm{~Pb} /{ }^{235} \mathrm{U}$} & \multicolumn{2}{|c|}{${ }^{206} \mathrm{~Pb} /{ }^{238} \mathrm{U}$} & \multicolumn{2}{|c|}{${ }^{207} \mathrm{~Pb} /{ }^{206} \mathrm{~Pb}$} & \multicolumn{2}{|c|}{${ }^{207} \mathrm{~Pb} /{ }^{235} \mathrm{U}$} & \multicolumn{2}{|c|}{${ }^{206} \mathrm{~Pb} /{ }^{238} \mathrm{U}$} & \multirow{2}{*}{ Concordance } \\
\hline & & & & Ratio & 1sigma & Ratio & 1sigma & Ratio & 1sigma & Age (Ma) & 1sigma & Age (Ma) & 1sigma & Age (Ma) & 1sigma & \\
\hline \multicolumn{17}{|c|}{91500} \\
\hline 1 & 36.6 & 104 & 0.35 & 0.0754 & 0.0019 & 1.8571 & 0.0449 & 0.1789 & 0.0020 & 1080 & 48.2 & 1066 & 16.0 & 1061 & 10.9 & $99 \%$ \\
\hline 2 & 35.3 & 101 & 0.35 & 0.0743 & 0.0020 & 1.8433 & 0.0514 & 0.1795 & 0.0022 & 1050 & 55.6 & 1061 & 18.4 & 1064 & 12.2 & $99 \%$ \\
\hline 3 & 33.9 & 96.8 & 0.35 & 0.0751 & 0.0020 & 1.8524 & 0.0515 & 0.1791 & 0.0021 & 1072 & 53.7 & 1064 & 18.4 & 1062 & 11.4 & $99 \%$ \\
\hline 4 & 34.6 & 97.9 & 0.35 & 0.0746 & 0.0019 & 1.8480 & 0.0529 & 0.1792 & 0.0022 & 1059 & 51.4 & 1063 & 18.9 & 1063 & 11.9 & $99 \%$ \\
\hline 5 & 34.9 & 99.4 & 0.35 & 0.0750 & 0.0021 & 1.8568 & 0.0500 & 0.1795 & 0.0023 & 1133 & 56.6 & 1066 & 17.8 & 1064 & 12.5 & $99 \%$ \\
\hline 6 & 34.8 & 99.1 & 0.35 & 0.0748 & 0.0020 & 1.8436 & 0.0485 & 0.1788 & 0.0027 & 1061 & 52.6 & 1061 & 17.3 & 1061 & 15.0 & $99 \%$ \\
\hline 7 & 25.3 & 71.0 & 0.36 & 0.0751 & 0.0020 & 1.8583 & 0.0528 & 0.1793 & 0.0022 & 1072 & 53.7 & 1066 & 18.8 & 1063 & 12.1 & $99 \%$ \\
\hline 8 & 24.8 & 71.3 & 0.35 & 0.0746 & 0.0024 & 1.8421 & 0.0650 & 0.1791 & 0.0024 & 1058 & 63.0 & 1061 & 23.2 & 1062 & 13.1 & $99 \%$ \\
\hline 9 & 25.8 & 74.6 & 0.35 & 0.0756 & 0.0018 & 1.8642 & 0.0435 & 0.1791 & 0.0022 & 1084 & 47.4 & 1068 & 15.5 & 1062 & 12.2 & $99 \%$ \\
\hline 10 & 26.9 & 75.9 & 0.35 & 0.0742 & 0.0018 & 1.8362 & 0.0472 & 0.1792 & 0.0021 & 1056 & 49.2 & 1059 & 16.9 & 1063 & 11.7 & $99 \%$ \\
\hline 11 & 25.8 & 74.0 & 0.35 & 0.0734 & 0.0019 & 1.8130 & 0.0441 & 0.1792 & 0.0023 & 1025 & 52.3 & 1050 & 15.9 & 1063 & 12.5 & $98 \%$ \\
\hline 12 & 26.0 & 73.9 & 0.35 & 0.0764 & 0.0019 & 1.8874 & 0.0490 & 0.1791 & 0.0023 & 1106 & 50.0 & 1077 & 17.3 & 1062 & 12.8 & $98 \%$ \\
\hline 13 & 26.3 & 75.1 & 0.35 & 0.0764 & 0.0019 & 1.8459 & 0.0556 & 0.1787 & 0.0023 & 1106 & 49.5 & 1062 & 19.8 & 1060 & 12.5 & $99 \%$ \\
\hline 14 & 26.8 & 75.5 & 0.35 & 0.0734 & 0.0020 & 1.8545 & 0.0759 & 0.1796 & 0.0022 & 1025 & 56.0 & 1065 & 27.0 & 1065 & 12.0 & $99 \%$ \\
\hline 15 & 20.2 & 56.9 & 0.36 & 0.0754 & 0.0026 & 1.8472 & 0.0654 & 0.1780 & 0.0026 & 1080 & 69 & 1062 & 23.3 & 1056 & 14.5 & $99 \%$ \\
\hline 16 & 19.7 & 56.7 & 0.35 & 0.0743 & 0.0025 & 1.8532 & 0.0610 & 0.1804 & 0.0029 & 1050 & 68 & 1065 & 21.7 & 1069 & 15.6 & $99 \%$ \\
\hline 17 & 19.7 & 56.4 & 0.35 & 0.0756 & 0.0022 & 1.8655 & 0.0544 & 0.1792 & 0.0027 & 1087 & 57 & 1069 & 19.3 & 1063 & 15.0 & $99 \%$ \\
\hline 18 & 19.0 & 55.6 & 0.34 & 0.0741 & 0.0021 & 1.8349 & 0.0552 & 0.1791 & 0.0028 & 1056 & 56 & 1058 & 19.8 & 1062 & 15.2 & $99 \%$ \\
\hline 19 & 19.1 & 55.2 & 0.35 & 0.0755 & 0.0022 & 1.8688 & 0.0580 & 0.1791 & 0.0027 & 1081 & 59 & 1070 & 20.6 & 1062 & 14.7 & $99 \%$ \\
\hline 20 & 18.8 & 54.5 & 0.34 & 0.0743 & 0.0025 & 1.8316 & 0.0592 & 0.1792 & 0.0027 & 1050 & 68 & 1057 & 21.3 & 1063 & 14.5 & $99 \%$ \\
\hline 21 & 17.3 & 49.8 & 0.35 & 0.0746 & 0.0023 & 1.8482 & 0.0605 & 0.1790 & 0.0025 & 1057 & 63 & 1063 & 21.6 & 1062 & 13.9 & $99 \%$ \\
\hline 22 & 16.9 & 48.9 & 0.35 & 0.0752 & 0.0025 & 1.8522 & 0.0607 & 0.1793 & 0.0025 & 1072 & 68 & 1064 & 21.6 & 1063 & 13.9 & $99 \%$ \\
\hline \multicolumn{17}{|c|}{ GJ-1 } \\
\hline 1 & 7.03 & 394 & 0.02 & 0.0573 & 0.0014 & 0.7775 & 0.0178 & 0.0983 & 0.0012 & 506 & 58 & 584 & 10.2 & 605 & 7.0 & $96 \%$ \\
\hline 2 & 11.5 & 639 & 0.02 & 0.0586 & 0.0012 & 0.7882 & 0.0170 & 0.0977 & 0.0012 & 554 & 42.6 & 590 & 9.7 & 601 & 6.9 & $98 \%$ \\
\hline 3 & 12.4 & 676 & 0.02 & 0.0617 & 0.0012 & 0.8195 & 0.0169 & 0.0961 & 0.0011 & 665 & 42.6 & 608 & 9.5 & 592 & 6.4 & $97 \%$ \\
\hline 4 & 7.09 & 399 & 0.02 & 0.0622 & 0.0013 & 0.8441 & 0.0192 & 0.0981 & 0.0013 & 683 & 42.6 & 621 & 10.6 & 603 & 7.9 & $97 \%$ \\
\hline 5 & 6.76 & 381 & 0.02 & 0.0608 & 0.0010 & 0.8173 & 0.0136 & 0.0975 & 0.0008 & 632 & 34.4 & 607 & 7.6 & 600 & 4.9 & $98 \%$ \\
\hline 6 & 6.97 & 395 & 0.02 & 0.0591 & 0.0010 & 0.7986 & 0.0138 & 0.0976 & 0.0009 & 572 & 2.8 & 596 & 7.8 & 601 & 5.4 & $99 \%$ \\
\hline 7 & 11.7 & 630 & 0.02 & 0.0598 & 0.0011 & 0.8033 & 0.0152 & 0.0975 & 0.0012 & 598 & 36.1 & 599 & 8.6 & 600 & 7.2 & $99 \%$ \\
\hline \multicolumn{17}{|c|}{ Plešovice } \\
\hline 1 & 106 & 1208 & 0.09 & 0.0511 & 0.0010 & 0.3800 & 0.0086 & 0.0538 & 0.0006 & 243 & 44.4 & 327 & 6.4 & 338 & 3.4 & $96 \%$ \\
\hline 2 & 527 & 3956 & 0.13 & 0.0516 & 0.0009 & 0.3912 & 0.0074 & 0.0548 & 0.0007 & 333 & 40.7 & 335 & 5.4 & 344 & 4.5 & $97 \%$ \\
\hline 3 & 92.9 & 1097 & 0.08 & 0.0536 & 0.0010 & 0.3986 & 0.0077 & 0.0538 & 0.0005 & 354 & 42.6 & 341 & 5.6 & 338 & 3.3 & $99 \%$ \\
\hline 4 & 91.7 & 1095 & 0.08 & 0.0540 & 0.0010 & 0.3968 & 0.0076 & 0.0531 & 0.0006 & 369 & 40.7 & 339 & 5.5 & 334 & 3.8 & $98 \%$ \\
\hline 5 & 71.5 & 865 & 0.08 & 0.0516 & 0.0009 & 0.3830 & 0.0089 & 0.0552 & 0.0006 & 333 & 40.7 & 329 & 6.5 & 346 & 3.7 & $94 \%$ \\
\hline 6 & 63.4 & 677 & 0.09 & 0.0535 & 0.0018 & 0.3964 & 0.0130 & 0.0537 & 0.0007 & 350 & 81 & 339 & 9.5 & 337 & 4.1 & $99 \%$ \\
\hline 7 & 54.8 & 598 & 0.09 & 0.0547 & 0.0014 & 0.4124 & 0.0109 & 0.0542 & 0.0006 & 467 & 56 & 351 & 7.8 & 341 & 3.8 & $97 \%$ \\
\hline
\end{tabular}


Table 2. LA-ICP-MS zircon U-Pb dating results of host granitic aplite and granites from two types of Cu-Mo mineralization in the NEYA.

\begin{tabular}{|c|c|c|c|c|c|c|c|c|c|c|c|c|c|c|c|c|}
\hline \multirow{2}{*}{ No. } & \multirow{2}{*}{ Th (ppm) } & \multirow{2}{*}{$\mathrm{U}(\mathrm{ppm})$} & \multirow{2}{*}{$\mathrm{Th} / \mathrm{U}$} & \multicolumn{2}{|c|}{${ }^{207} \mathrm{~Pb} /{ }^{206} \mathrm{~Pb}$} & \multicolumn{2}{|c|}{${ }^{207} \mathrm{~Pb} /{ }^{235} \mathrm{U}$} & \multicolumn{2}{|c|}{${ }^{206} \mathrm{~Pb} /{ }^{238} \mathrm{U}$} & \multicolumn{2}{|c|}{${ }^{207} \mathrm{~Pb} /{ }^{206} \mathrm{~Pb}$} & \multicolumn{2}{|c|}{${ }^{207} \mathrm{~Pb} /{ }^{235} \mathrm{U}$} & \multicolumn{2}{|c|}{${ }^{206} \mathrm{~Pb} /{ }^{238} \mathrm{U}$} & \multirow{2}{*}{ Concordance } \\
\hline & & & & Ratio & 1sigma & Ratio & 1sigma & Ratio & 1sigma & Age (Ma) & 1sigma & Age (Ma) & 1sigma & Age (Ma) & 1sigma & \\
\hline \multicolumn{17}{|c|}{ Sample DC16-65 } \\
\hline DC16-65-01 & 791 & 1852 & 0.43 & 0.0504 & 0.0022 & 0.2379 & 0.0096 & 0.0346 & 0.0004 & 216.7 & 106 & 216.7 & 7.9 & 219.6 & 2.2 & $98 \%$ \\
\hline DC16-65-02 & 3111 & 8616 & 0.36 & 0.0487 & 0.0009 & 0.2327 & 0.0054 & 0.0345 & 0.0006 & 200.1 & 38.0 & 212.4 & 4.5 & 218.7 & 3.8 & $97 \%$ \\
\hline DC16-65-03 & 1020 & 2615 & 0.39 & 0.0505 & 0.0008 & 0.2444 & 0.0051 & 0.0351 & 0.0006 & 220.4 & 34.3 & 222.0 & 4.2 & 222.6 & 3.7 & $99 \%$ \\
\hline DC16-65-04 & 2350 & 4511 & 0.52 & 0.0500 & 0.0026 & 0.2465 & 0.0149 & 0.0343 & 0.0005 & 194.5 & 122 & 223.8 & 12.1 & 217.5 & 2.9 & $97 \%$ \\
\hline DC16-65-05 & 649 & 1109 & 0.59 & 0.0477 & 0.0014 & 0.2252 & 0.0077 & 0.0340 & 0.0004 & 87.1 & 76.8 & 206.2 & 6.4 & 215.5 & 2.6 & $95 \%$ \\
\hline DC16-65-06 & 1055 & 1898 & 0.56 & 0.0487 & 0.0009 & 0.2313 & 0.0050 & 0.0343 & 0.0004 & 200.1 & 38.0 & 211.2 & 4.1 & 217.3 & 2.4 & $97 \%$ \\
\hline DC16-65-07 & 648 & 1901 & 0.34 & 0.0507 & 0.0009 & 0.2381 & 0.0051 & 0.0340 & 0.0005 & 227.8 & 71.3 & 216.9 & 4.2 & 215.4 & 3.2 & $99 \%$ \\
\hline DC16-65-08 & 1930 & 4287 & 0.45 & 0.0505 & 0.0014 & 0.2442 & 0.0087 & 0.0343 & 0.0006 & 216.7 & 64.8 & 221.8 & 7.1 & 217.7 & 3.8 & $98 \%$ \\
\hline DC16-65-09 & 1607 & 2924 & 0.55 & 0.0505 & 0.0009 & 0.2401 & 0.0043 & 0.0344 & 0.0003 & 220.4 & 36.1 & 218.5 & 3.6 & 217.7 & 1.7 & $99 \%$ \\
\hline DC16-65-10 & 1570 & 3795 & 0.41 & 0.0501 & 0.0021 & 0.2402 & 0.0098 & 0.0342 & 0.0006 & 198.2 & 100 & 218.5 & 8.1 & 217.0 & 3.7 & $99 \%$ \\
\hline DC16-65-11 & 881 & 1617 & 0.54 & 0.0532 & 0.0009 & 0.2534 & 0.0054 & 0.0344 & 0.0005 & 344.5 & 37.0 & 229.3 & 4.4 & 217.9 & 3.1 & $94 \%$ \\
\hline DC16-65-12 & 1308 & 2403 & 0.54 & 0.0504 & 0.0015 & 0.2356 & 0.0068 & 0.0340 & 0.0006 & 213.0 & 70.4 & 214.8 & 5.6 & 215.8 & 3.5 & $99 \%$ \\
\hline DC16-65-13 & 1596 & 2576 & 0.62 & 0.0472 & 0.0024 & 0.2233 & 0.0115 & 0.0344 & 0.0003 & 57.5 & 119 & 204.6 & 9.6 & 217.8 & 2.0 & $93 \%$ \\
\hline DC16-65-14 & 1532 & 2983 & 0.51 & 0.0487 & 0.0039 & 0.2207 & 0.0170 & 0.0344 & 0.0004 & 200.1 & 122 & 202.5 & 14.1 & 218.0 & 2.3 & $92 \%$ \\
\hline DC16-65-15 & 1053 & 1392 & 0.76 & 0.0509 & 0.0036 & 0.2297 & 0.0169 & 0.0339 & 0.0005 & 239.0 & 165 & 209.9 & 14.0 & 215.0 & 3.3 & $97 \%$ \\
\hline DC16-65-16 & 1208 & 3058 & 0.39 & 0.0495 & 0.0020 & 0.2297 & 0.0106 & 0.0336 & 0.0005 & 172.3 & 129 & 209.9 & 8.8 & 212.8 & 2.8 & $98 \%$ \\
\hline \multicolumn{17}{|c|}{ Sample DC16-68 } \\
\hline DC16-68-01 & 387 & 955 & 0.41 & 0.0506 & 0.0011 & 0.2482 & 0.0058 & 0.0354 & 0.0004 & 220.4 & 80.5 & 225.2 & 4.7 & 224.0 & 2.4 & $99 \%$ \\
\hline DC16-68-02 & 469 & 1101 & 0.43 & 0.0504 & 0.0011 & 0.2373 & 0.0052 & 0.0342 & 0.0004 & 213.0 & 49.1 & 216.2 & 4.3 & 217.0 & 2.6 & $99 \%$ \\
\hline DC16-68-03 & 411 & 991 & 0.42 & 0.0512 & 0.0010 & 0.2574 & 0.0052 & 0.0364 & 0.0004 & 250.1 & 41.7 & 232.6 & 4.2 & 230.4 & 2.4 & $99 \%$ \\
\hline DC16-68-04 & 423 & 1164 & 0.36 & 0.0507 & 0.0010 & 0.2479 & 0.0050 & 0.0356 & 0.0004 & 233.4 & 48.1 & 224.8 & 4.1 & 225.2 & 2.3 & $99 \%$ \\
\hline DC16-68-05 & 252 & 685 & 0.37 & 0.0506 & 0.0012 & 0.2502 & 0.0054 & 0.0358 & 0.0004 & 233.4 & 51.8 & 226.8 & 4.4 & 227.0 & 2.4 & $99 \%$ \\
\hline DC16-68-06 & 295 & 790 & 0.37 & 0.0508 & 0.0011 & 0.2590 & 0.0057 & 0.0370 & 0.0005 & 231.6 & 47.2 & 233.9 & 4.6 & 234.5 & 2.9 & $99 \%$ \\
\hline \multicolumn{17}{|c|}{ Sample DC16-68 } \\
\hline DC16-68-07 & 445 & 1249 & 0.36 & 0.0508 & 0.0010 & 0.2543 & 0.0049 & 0.0362 & 0.0004 & 231.6 & 44.4 & 230.1 & 4.0 & 229.4 & 2.3 & $99 \%$ \\
\hline DC16-68-08 & 518 & 880 & 0.59 & 0.0505 & 0.0012 & 0.2382 & 0.0053 & 0.0343 & 0.0004 & 216.7 & 86.1 & 217.0 & 4.3 & 217.3 & 2.6 & $99 \%$ \\
\hline DC16-68-09 & 435 & 1341 & 0.32 & 0.0504 & 0.0019 & 0.2436 & 0.0097 & 0.0350 & 0.0005 & 213.0 & 87.0 & 221.3 & 7.9 & 221.8 & 3.2 & $99 \%$ \\
\hline DC16-68-10 & 506 & 1038 & 0.49 & 0.0505 & 0.0012 & 0.2399 & 0.0056 & 0.0344 & 0.0004 & 220.4 & 55.5 & 218.3 & 4.6 & 217.9 & 2.3 & $99 \%$ \\
\hline DC16-68-11 & 312 & 789 & 0.40 & 0.0577 & 0.0011 & 0.6642 & 0.0158 & 0.0828 & 0.0011 & 516.7 & 45.4 & 517.2 & 9.6 & 513.0 & 6.8 & $99 \%$ \\
\hline DC16-68-12 & 364 & 532 & 0.68 & 0.0574 & 0.0012 & 0.6440 & 0.0131 & 0.0816 & 0.0012 & 505.6 & 44.4 & 504.7 & 8.1 & 505.7 & 7.0 & $99 \%$ \\
\hline DC16-68-13 & 350 & 572 & 0.61 & 0.0509 & 0.0014 & 0.2562 & 0.0070 & 0.0365 & 0.0004 & 235.3 & 63.0 & 231.6 & 5.6 & 231.4 & 2.5 & $99 \%$ \\
\hline DC16-68-14 & 486 & 1047 & 0.46 & 0.0509 & 0.0011 & 0.2562 & 0.0055 & 0.0365 & 0.0004 & 239.0 & 50.0 & 231.6 & 4.5 & 230.9 & 2.5 & $99 \%$ \\
\hline DC16-68-17 & 749 & 1880 & 0.40 & 0.0506 & 0.0011 & 0.2360 & 0.0048 & 0.0339 & 0.0004 & 220.4 & 80.5 & 215.2 & 4.0 & 215.2 & 2.6 & $99 \%$ \\
\hline DC16-68-15 & 328 & 958 & 0.34 & 0.0504 & 0.0011 & 0.2469 & 0.0053 & 0.0356 & 0.0004 & 213.0 & 51.8 & 224.0 & 4.3 & 225.7 & 2.7 & $99 \%$ \\
\hline DC16-68-16 & 756 & 1948 & 0.39 & 0.0522 & 0.0009 & 0.2559 & 0.0047 & 0.0355 & 0.0004 & 294.5 & 38.9 & 231.4 & 3.8 & 224.7 & 2.2 & $97 \%$ \\
\hline DC16-68-20 & 381 & 933 & 0.41 & 0.0505 & 0.0013 & 0.2428 & 0.0061 & 0.0351 & 0.0005 & 220.4 & 54.6 & 220.7 & 5.0 & 222.2 & 3.3 & $99 \%$ \\
\hline DC16-68-21 & 531 & 1033 & 0.51 & 0.0502 & 0.0012 & 0.2345 & 0.0058 & 0.0339 & 0.0005 & 205.6 & 57.4 & 213.9 & 4.8 & 215.2 & 2.8 & $99 \%$ \\
\hline DC16-68-22 & 737 & 1335 & 0.55 & 0.0503 & 0.0010 & 0.2357 & 0.0052 & 0.0340 & 0.0004 & 209.3 & 46.3 & 214.9 & 4.3 & 215.6 & 2.7 & $99 \%$ \\
\hline
\end{tabular}


Table 2. Cont

\begin{tabular}{|c|c|c|c|c|c|c|c|c|c|c|c|c|c|c|c|c|}
\hline \multirow{2}{*}{ No. } & \multirow{2}{*}{ Th (ppm) } & \multirow{2}{*}{$\mathrm{U}(\mathrm{ppm})$} & \multirow{2}{*}{$\mathrm{Th} / \mathrm{U}$} & \multicolumn{2}{|c|}{${ }^{207} \mathrm{~Pb} /{ }^{206} \mathrm{~Pb}$} & \multicolumn{2}{|c|}{${ }^{207} \mathrm{~Pb} /{ }^{235} \mathrm{U}$} & \multicolumn{2}{|c|}{${ }^{206} \mathrm{~Pb} /{ }^{238} \mathrm{U}$} & \multicolumn{2}{|c|}{${ }^{207} \mathrm{~Pb} /{ }^{206} \mathrm{~Pb}$} & \multicolumn{2}{|c|}{${ }^{207} \mathrm{~Pb} /{ }^{235} \mathrm{U}$} & \multicolumn{2}{|c|}{${ }^{206} \mathrm{~Pb} /{ }^{238} \mathrm{U}$} & \multirow{2}{*}{ Concordance } \\
\hline & & & & Ratio & 1sigma & Ratio & 1sigma & Ratio & 1sigma & Age (Ma) & 1sigma & Age (Ma) & 1sigma & Age (Ma) & 1sigma & \\
\hline DC16-68-23 & 714 & 922 & 0.77 & 0.0508 & 0.0012 & 0.2440 & 0.0058 & 0.0349 & 0.0004 & 231.6 & 55.5 & 221.7 & 4.7 & 221.4 & 2.7 & $99 \%$ \\
\hline DC16-68-24 & 758 & 1907 & 0.40 & 0.0508 & 0.0009 & 0.2364 & 0.0052 & 0.0336 & 0.0005 & 231.6 & 71.3 & 215.5 & 4.3 & 213.3 & 3.0 & $99 \%$ \\
\hline DC16-68-25 & 668 & 1042 & 0.64 & 0.0506 & 0.0010 & 0.2436 & 0.0049 & 0.0351 & 0.0005 & 220.4 & 44.4 & 221.3 & 4.0 & 222.1 & 3.3 & $99 \%$ \\
\hline DC16-68-26 & 439 & 1264 & 0.35 & 0.0506 & 0.0011 & 0.2396 & 0.0054 & 0.0344 & 0.0005 & 220.4 & 51.8 & 218.1 & 4.5 & 218.2 & 3.0 & $99 \%$ \\
\hline DC16-68-27 & 504 & 1492 & 0.34 & 0.0499 & 0.0011 & 0.2391 & 0.0068 & 0.0353 & 0.0009 & 187.1 & 19.4 & 217.7 & 5.6 & 223.7 & 5.7 & $97 \%$ \\
\hline DC16-68-28 & 1045 & 1424 & 0.73 & 0.0495 & 0.0011 & 0.2354 & 0.0061 & 0.0347 & 0.0008 & 172.3 & 50.0 & 214.6 & 5.0 & 219.9 & 4.9 & $97 \%$ \\
\hline DC16-68-29 & 429 & 1163 & 0.37 & 0.0523 & 0.0015 & 0.2536 & 0.0074 & 0.0354 & 0.0008 & 298.2 & 66.7 & 229.5 & 6.0 & 224.5 & 4.9 & $97 \%$ \\
\hline DC16-68-30 & 550 & 1474 & 0.37 & 0.0488 & 0.0013 & 0.2348 & 0.0079 & 0.0350 & 0.0009 & 200.1 & 66.7 & 214.1 & 6.5 & 221.9 & 5.6 & $96 \%$ \\
\hline \multicolumn{17}{|c|}{ Sample GZ16-40 } \\
\hline GZ16-40-02 & 462 & 723 & 0.64 & 0.0504 & 0.0012 & 0.2390 & 0.0059 & 0.0343 & 0.0004 & 213.0 & 49.1 & 217.6 & 4.9 & 217.6 & 2.8 & $99 \%$ \\
\hline GZ16-40-03 & 499 & 1097 & 0.46 & 0.0505 & 0.0015 & 0.2432 & 0.0079 & 0.0348 & 0.0005 & 216.7 & 68.5 & 221.1 & 6.5 & 220.4 & 3.3 & $99 \%$ \\
\hline GZ16-40-04 & 242 & 530 & 0.46 & 0.0506 & 0.0013 & 0.2554 & 0.0069 & 0.0366 & 0.0005 & 233.4 & 61.1 & 231.0 & 5.6 & 231.6 & 2.8 & $99 \%$ \\
\hline GZ16-40-05 & 468 & 851 & 0.55 & 0.0505 & 0.0011 & 0.2356 & 0.0054 & 0.0338 & 0.0003 & 216.7 & 84.2 & 214.8 & 4.4 & 214.1 & 2.1 & $99 \%$ \\
\hline GZ16-40-07 & 193 & 387 & 0.50 & 0.0506 & 0.0017 & 0.2403 & 0.0081 & 0.0346 & 0.0004 & 220.4 & 81.5 & 218.7 & 6.6 & 219.3 & 2.7 & $99 \%$ \\
\hline GZ16-40-08 & 280 & 502 & 0.56 & 0.0504 & 0.0014 & 0.2425 & 0.0067 & 0.0349 & 0.0005 & 213.0 & 64.8 & 220.5 & 5.4 & 221.0 & 3.4 & $99 \%$ \\
\hline GZ16-40-09 & 222 & 516 & 0.43 & 0.0504 & 0.0013 & 0.2357 & 0.0059 & 0.0340 & 0.0004 & 213.0 & 59.2 & 214.9 & 4.8 & 215.8 & 2.7 & $99 \%$ \\
\hline GZ16-40-10 & 499 & 1144 & 0.44 & 0.0506 & 0.0011 & 0.2408 & 0.0053 & 0.0346 & 0.0004 & 220.4 & 82.4 & 219.1 & 4.4 & 219.4 & 2.8 & $99 \%$ \\
\hline GZ16-40-11 & 248 & 635 & 0.39 & 0.0507 & 0.0017 & 0.2330 & 0.0067 & 0.0338 & 0.0006 & 227.8 & 79.6 & 212.7 & 5.5 & 214.1 & 3.8 & $99 \%$ \\
\hline GZ16-40-12 & 644 & 1085 & 0.59 & 0.0505 & 0.0011 & 0.2377 & 0.0054 & 0.0341 & 0.0004 & 220.4 & 82.4 & 216.6 & 4.4 & 216.4 & 2.4 & $99 \%$ \\
\hline GZ16-40-14 & 179 & 350 & 0.51 & 0.0507 & 0.0017 & 0.2508 & 0.0082 & 0.0363 & 0.0006 & 227.8 & 79.6 & 227.2 & 6.7 & 230.0 & 3.7 & $98 \%$ \\
\hline GZ16-40-15 & 475 & 1232 & 0.39 & 0.0514 & 0.0012 & 0.2577 & 0.0061 & 0.0363 & 0.0004 & 257.5 & 55.5 & 232.8 & 4.9 & 230.1 & 2.5 & $98 \%$ \\
\hline GZ16-40-16 & 319 & 683 & 0.47 & 0.0510 & 0.0012 & 0.2563 & 0.0064 & 0.0366 & 0.0006 & 239.0 & 58.3 & 231.7 & 5.1 & 231.9 & 3.6 & $99 \%$ \\
\hline GZ16-40-17 & 423 & 780 & 0.54 & 0.0504 & 0.0012 & 0.2335 & 0.0052 & 0.0337 & 0.0004 & 213.0 & 83.3 & 213.1 & 4.3 & 213.9 & 2.5 & $99 \%$ \\
\hline GZ16-40-18 & 508 & 1067 & 0.48 & 0.0508 & 0.0012 & 0.2438 & 0.0055 & 0.0348 & 0.0004 & 231.6 & 53.7 & 221.5 & 4.5 & 220.7 & 2.4 & $99 \%$ \\
\hline GZ16-40-19 & 282 & 489 & 0.58 & 0.0518 & 0.0014 & 0.2621 & 0.0073 & 0.0364 & 0.0004 & 279.7 & 61.1 & 236.3 & 5.8 & 230.4 & 2.4 & $97 \%$ \\
\hline GZ16-40-20 & 385 & 761 & 0.51 & 0.0541 & 0.0012 & 0.3524 & 0.0124 & 0.0471 & 0.0013 & 372.3 & 54.6 & 306.6 & 9.3 & 296.8 & 7.8 & $96 \%$ \\
\hline GZ16-40-21 & 758 & 868 & 0.87 & 0.0506 & 0.0011 & 0.2601 & 0.0058 & 0.0372 & 0.0004 & 233.4 & 51.8 & 234.7 & 4.7 & 235.6 & 2.2 & $99 \%$ \\
\hline GZ16-40-22 & 231 & 509 & 0.45 & 0.0517 & 0.0015 & 0.2628 & 0.0075 & 0.0368 & 0.0004 & 272.3 & 69.4 & 236.9 & 6.0 & 233.3 & 2.6 & $98 \%$ \\
\hline GZ16-40-23 & 310 & 573 & 0.54 & 0.0508 & 0.0013 & 0.2587 & 0.0071 & 0.0369 & 0.0005 & 231.6 & 59.3 & 233.6 & 5.8 & 233.4 & 2.8 & $99 \%$ \\
\hline GZ16-40-24 & 488 & 1171 & 0.42 & 0.0499 & 0.0022 & 0.2348 & 0.0117 & 0.0337 & 0.0005 & 190.8 & 100 & 214.2 & 9.6 & 213.4 & 3.2 & $99 \%$ \\
\hline GZ16-40-25 & 206 & 379 & 0.54 & 0.0510 & 0.0019 & 0.2677 & 0.0093 & 0.0382 & 0.0004 & 239.0 & 85.2 & 240.9 & 7.4 & 241.7 & 2.7 & $99 \%$ \\
\hline GZ16-40-26 & 416 & 581 & 0.72 & 0.0505 & 0.0015 & 0.2409 & 0.0073 & 0.0347 & 0.0005 & 220.4 & 66.7 & 219.2 & 6.0 & 219.6 & 3.2 & $99 \%$ \\
\hline GZ16-40-27 & 644 & 913 & 0.70 & 0.0509 & 0.0012 & 0.2575 & 0.0064 & 0.0366 & 0.0004 & 235.3 & 55.5 & 232.7 & 5.2 & 231.6 & 2.5 & $99 \%$ \\
\hline GZ16-40-29 & 257 & 679 & 0.38 & 0.0511 & 0.0012 & 0.2605 & 0.0084 & 0.0367 & 0.0007 & 242.7 & 55.5 & 235.1 & 6.7 & 232.2 & 4.4 & $98 \%$ \\
\hline GZ16-40-30 & 532 & 827 & 0.64 & 0.0508 & 0.0012 & 0.2386 & 0.0057 & 0.0342 & 0.0005 & 231.6 & 53.7 & 217.3 & 4.7 & 216.5 & 3.1 & $99 \%$ \\
\hline
\end{tabular}




\section{Results}

\subsection{Zircon $\mathrm{U}-\mathrm{Pb}$ Ages}

Zircon grains separated from granitic aplite (DC16-65; Figure 2) are mainly colorless, euhedral to subhedral shape, with lengths varying from 50 to $120 \mu \mathrm{m}$ and the length to width ratios ranging from about 2:1 to 1:1. In the CL images, these zircons show obvious oscillatory zoning (Figure 5a), indicative of the igneous origin [46,47]. Sixteen U-Pb analyses on 16 zircon grains were obtained. The concentrations of Th and U of these zircon grains vary from 648 to 3111 ppm and 1109 to 8616 ppm, respectively, with $\mathrm{Th} / \mathrm{U}$ ratios varying from 0.34 to 0.76 (Table 2 ). All analyses are concordant within analytical errors and yield a concordia age of 217.3 $\pm 1.4 \mathrm{Ma}(\mathrm{MSWD}=0.22, \mathrm{n}=16)$, with a weighted mean age of $217.3 \pm 1.3 \mathrm{Ma}(\mathrm{MSWD}=0.50, \mathrm{n}=16)($ Figure $6 \mathrm{a}, \mathrm{b})$, representing the formation age of the granitic aplite.
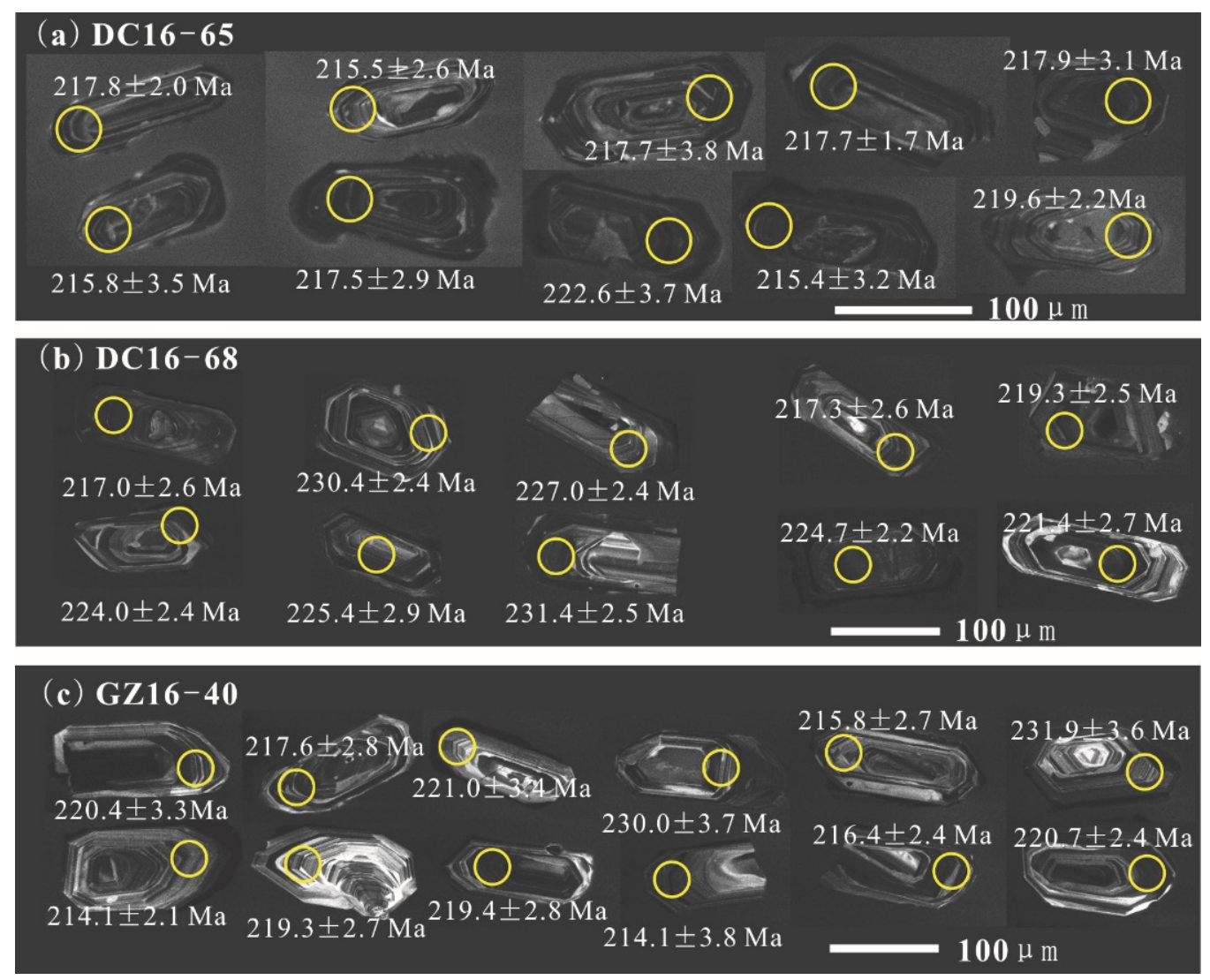

Figure 5. Cathodoluminescence (CL) images of zircons from granitic host rocks of two types of $\mathrm{Cu}-\mathrm{Mo}$ mineralization at the NEYA. The yellow circle represents the location of LA-ICP-MS U-Pb dating. (a) The CL images of representative zircon grains from sample DC16-65. (b) The CL images of representative zircon grains from sample DC16-68. (c) The CL images of representative zircon grains from sample GZ16-40.

Zircon grains from sample DC16-68 (Figure 2) are transparent, euhedral. They have grain sizes ranging from 70 to $110 \mu \mathrm{m}$ in length and from 50 to $80 \mu \mathrm{m}$ in width, with length/width ratios of 2:1-1:1. CL images show that most of the zircon grains exhibit obvious oscillatory zoning (Figure 5b), indicating the igneous origin [46,47]. The Th/ $\mathrm{U}$ ratios of zircons vary from 0.32 to 0.77 , which further supports the igneous origin [48]. Thirty $\mathrm{U}-\mathrm{Pb}$ ages were obtained on 30 zircon grains, of which two grains have ${ }^{206} \mathrm{~Pb} /{ }^{238} \mathrm{U}$ ages of $505.7 \pm 7.0 \mathrm{Ma}$ and $513.0 \pm 6.8 \mathrm{Ma}$, and thirteen zircons have ${ }^{206} \mathrm{~Pb} /{ }^{238} \mathrm{U}$ ages ranging from $223.7 \pm 5.7 \mathrm{Ma}$ to $234.5 \pm 2.9 \mathrm{Ma}$, and fifteen have ${ }^{206} \mathrm{~Pb} /{ }^{238} \mathrm{U}$ ages of $222.2 \pm 3.3 \mathrm{Ma}$ to $213.3 \pm 3 \mathrm{Ma}$ (Table 2). The older zircon grains (513.0 $\pm 6.8 \mathrm{Ma}$ to $223.7 \pm 5.7 \mathrm{Ma}$ ) are interpreted as 
inherited or xenocrystic grains that were captured during the ascent of magma. The remaining fifteen zircon grains yield a concordia age of $218.0 \pm 1.5 \mathrm{Ma}(\mathrm{MSWD}=1.5, \mathrm{n}=15)$, with a weighted mean ${ }^{206} \mathrm{~Pb} /{ }^{238} \mathrm{U}$ age of $218.1 \pm 1.5 \mathrm{Ma}(\mathrm{MSWD}=0.92, \mathrm{n}=15$ ) (Figure $6 \mathrm{c}, \mathrm{d})$, representing the formation age of this sample.
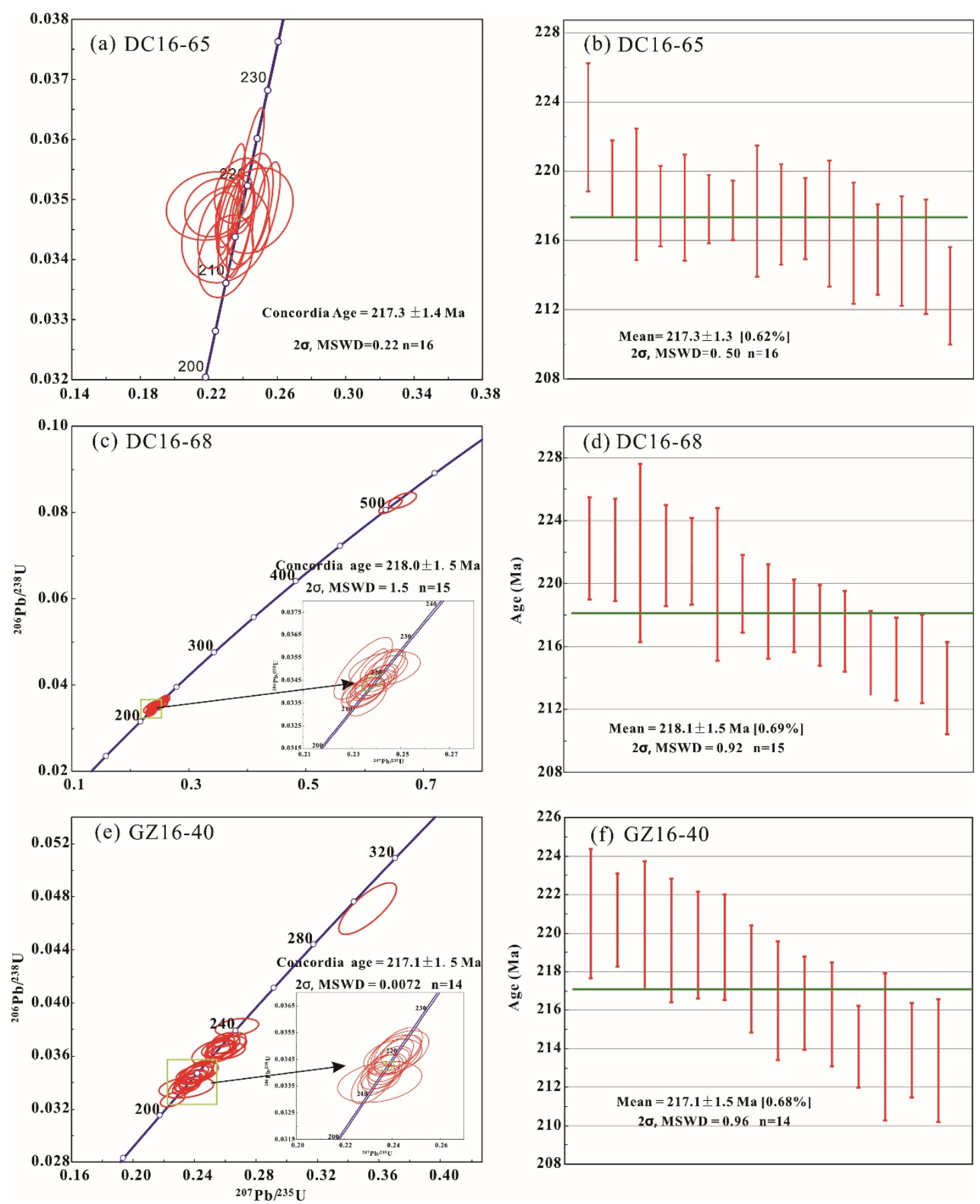

Figure 6. Zircon $\mathrm{U}-\mathrm{Pb}$ concordia and ${ }^{206} \mathrm{~Pb} /{ }^{238} \mathrm{U}$ weighted mean age diagrams for the host rocks of two types of $\mathrm{Cu}-\mathrm{Mo}$ mineralization in the NEYA. (a) the zircon U-Pb concordia and (b) ${ }^{206} \mathrm{~Pb} /{ }^{238} \mathrm{U}$ weighted mean age diagrams of sample DC16-65. (c) the zircon U-Pb concordia and (d) ${ }^{206} \mathrm{~Pb} /{ }^{238} \mathrm{U}$ weighted mean age diagrams of sample DC16-68. (e) the zircon U-Pb concordia and (f) ${ }^{206} \mathrm{~Pb} /{ }^{238} \mathrm{U}$ weighted mean age diagrams of sample GZ16-40. 
Zircon grains from sample GZ16-40 (Figure 3) are colorless and transparent, with euhedral and prismatic morphology. They are mostly ranging from 80 to $120 \mu \mathrm{m}$ in length and from 60 to $80 \mu \mathrm{m}$ in width, with the length/width ratio of 2:1-1.2:1.

These zircon grains generally show clear oscillatory zoning (Figure $5 c$ ) and high $\mathrm{Th} / \mathrm{U}$ ratios (0.38-0.87) (Table 2), indicating the magmatic origin [46-48]. Twenty-six U-Pb ages were obtained on 26 zircon grains from this sample, and their ${ }^{206} \mathrm{~Pb} /{ }^{238} \mathrm{U}$ ages vary from $296.8 \pm 7.8 \mathrm{Ma}$ to $213.4 \pm 3.2 \mathrm{Ma}$ (Table 2). Among these zircons, the oldest ${ }^{206} \mathrm{~Pb} /{ }^{238} \mathrm{U}$ age is $296.8 \pm 7.8 \mathrm{Ma}$, eleven zircon grains have ${ }^{206} \mathrm{~Pb} / 238 \mathrm{U}$ age of $241.7 \pm 2.7 \mathrm{Ma}$ to $230.0 \pm 3.7 \mathrm{Ma}$ with an average mean age of $233.1 \pm 2.3 \mathrm{Ma}$. The remaining fourteen zircon grains have ${ }^{206} \mathrm{~Pb} /{ }^{238} \mathrm{U}$ ages ranging from $221.0 \pm 3.4 \mathrm{Ma}$ to $213.4 \pm 3.2 \mathrm{Ma}$, with a concordia age of $217.1 \pm 0.75 \mathrm{Ma}$ (Figure 6e). It agrees well with the weighted mean ages of $217.1 \pm 1.5 \mathrm{Ma}(\mathrm{MSWD}=0.96, \mathrm{n}=14$ ), which represents the formation age of this sample (Figure $6 \mathrm{f}$ ).

\subsection{Molybdenite Re-Os Ages}

The Re-Os analytical results for seven molybdenite samples are given in Table 3. Molybdenites separated from type I Cu-Mo mineralization have highly variable total $\mathrm{Re},{ }^{187} \mathrm{Re}$ and ${ }^{187} \mathrm{Os}$ contents, ranging from 12.77 to $111.1 \mathrm{ppm}, 8.028$ to $69.84 \mathrm{ppm}$, and 28.8 to $259.1 \mathrm{ppb}$, respectively (Table 3). The Re-Os model ages range from $214.8 \pm 3.3 \mathrm{Ma}$ to $222.3 \pm 3.9 \mathrm{Ma}$ with an average mean age of $217.7 \pm 3.6 \mathrm{Ma}$. Four molybdenite samples yield a ${ }^{187} \mathrm{Re}^{-187} \mathrm{Os}$ isochron age of $220 \pm 18 \mathrm{Ma}$, which is coherent well with the weight mean age of $217.7 \pm 3.6 \mathrm{Ma}$ (Figure 7a,b).

Table 3. Re-Os isotopic data of molybdenites from two types of $\mathrm{Cu}-\mathrm{Mo}$ mineralization in the NEYA.

\begin{tabular}{|c|c|c|c|c|c|c|c|c|c|c|c|}
\hline \multirow{2}{*}{ Sample No. } & \multirow{2}{*}{ Weight (g) } & \multicolumn{2}{|c|}{$\operatorname{Re}(\mathrm{ppm})$} & \multicolumn{2}{|c|}{ Normal Os (ppb) } & \multicolumn{2}{|c|}{${ }^{187} \operatorname{Re}(\mathrm{ppm})$} & \multicolumn{2}{|c|}{${ }^{187}$ Os (ppb) } & \multicolumn{2}{|c|}{ Age (Ma) } \\
\hline & & Measured & $2 \sigma$ & Measured & $2 \sigma$ & Measured & $2 \sigma$ & Measured & $2 \sigma$ & Age & $2 \sigma$ \\
\hline \multicolumn{12}{|c|}{ Type I Cu-Mo mineralization } \\
\hline DC16-67 & 0.08904 & 12.77 & 0.12 & 0.5317 & 0.0701 & 8.028 & 0.072 & 28.8 & 0.2 & 214.8 & 3.3 \\
\hline DC16-68 & 0.01039 & 111.1 & 1.4 & 0.002763 & 0.035358 & 69.84 & 0.91 & 259.1 & 1.6 & 222.3 & 3.9 \\
\hline DC16-69 & 0.01032 & 109.6 & 1.1 & 0.002755 & 0.062690 & 68.90 & 0.68 & 251.8 & 1.6 & 219.0 & 3.4 \\
\hline DC16-70 & 0.01024 & 101.5 & 1.0 & 0.3558 & 0.0640 & 63.78 & 0.62 & 229.0 & 1.5 & 215.2 & 3.3 \\
\hline \multicolumn{12}{|c|}{ Type II Cu-Mo mineralization } \\
\hline GZ16-35 & 0.04323 & 7.983 & 0.072 & 0.3323 & 0.0438 & 5.018 & 0.045 & 17.81 & 0.13 & 212.6 & 3.2 \\
\hline GZ16-40 & 0.04012 & 10.05 & 0.12 & 0.5167 & 0.0525 & 6.317 & 0.077 & 22.50 & 0.15 & 213.4 & 3.6 \\
\hline GZ16-46 & 0.05900 & 10.40 & 0.09 & 0.1346 & 0.0178 & 6.536 & 0.055 & 22.89 & 0.16 & 209.8 & 3.1 \\
\hline
\end{tabular}

The total $\mathrm{Re},{ }^{187} \mathrm{Re}$ and ${ }^{187} \mathrm{Os}$ concentrations of molybdenite collected from type II Cu-Mo mineralization vary from 7.983 to $10.40 \mathrm{ppm}, 5.018$ to $6.536 \mathrm{ppm}$, and 17.81 to $22.89 \mathrm{ppb}$, respectively (Table 3). Three molybdenite samples yield relatively tight Re-Os model age of 212.6 $\pm 3.4 \mathrm{Ma}$, $213.4 \pm 3.6 \mathrm{Ma}$, and $209.8 \pm 3.1 \mathrm{Ma}$, with an average mean age of $211.8 \pm 4.7 \mathrm{Ma}$ (Figure $7 \mathrm{~d}$ ). This age agrees well with the ${ }^{187} \mathrm{Re}^{-187} \mathrm{Os}$ isochron age of $205 \pm 11 \mathrm{Ma}$ (MSWD $=2.8$ ) within error (Figure 7c), and represents the crystallization age of molybdenite in this type of $\mathrm{Cu}-\mathrm{Mo}$ mineralization. 

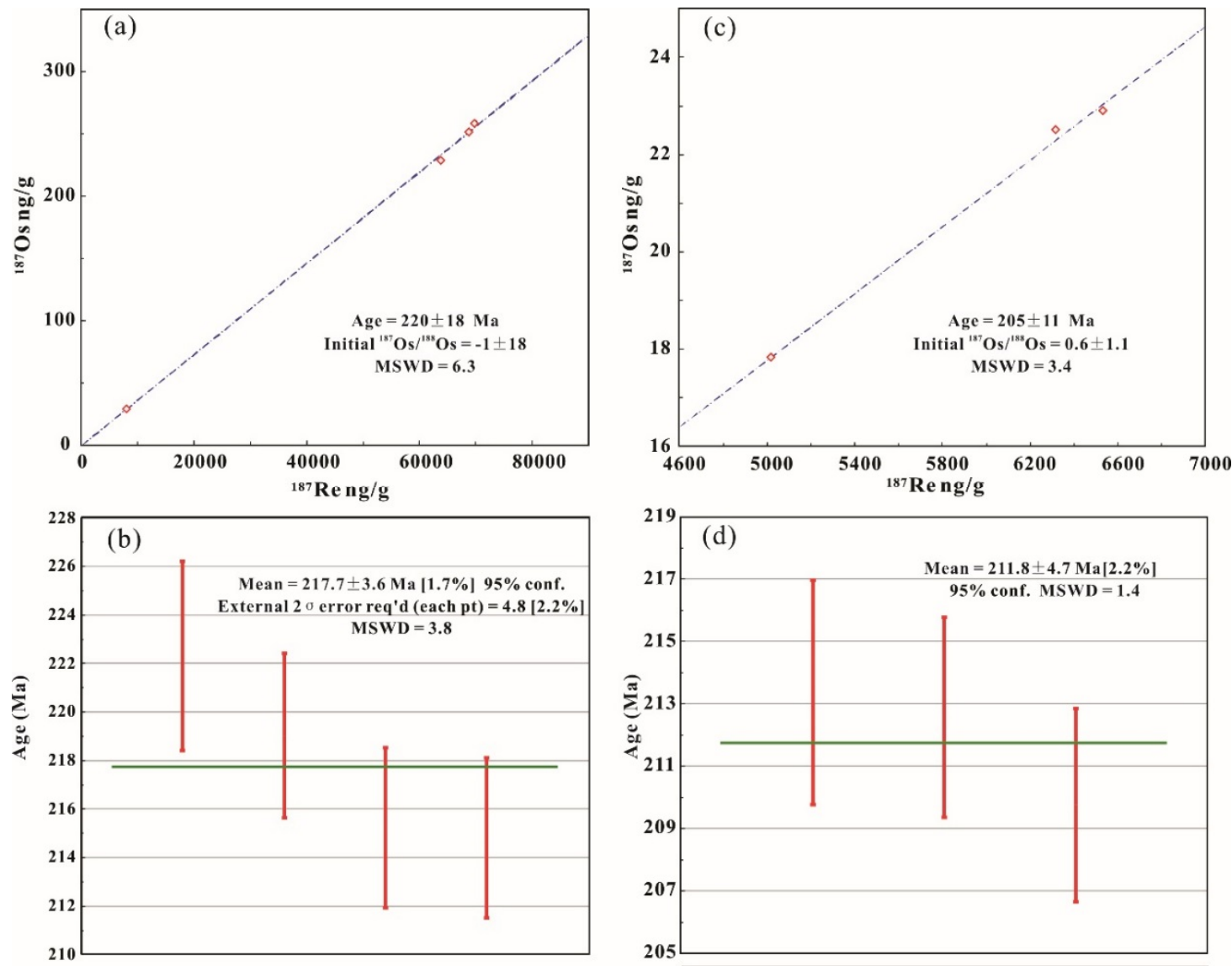

Figure 7. Re-Os isochron and average mean model age diagrams for molybdenites from two types of Cu-Mo mineralization in the NEYA. (a) the Re-Os isochron and (b) average mean model age diagrams of molybdenite from type I Cu-Mo mineralization. (c) the Re-Os isochron and (d) average mean model age diagrams of molybdenite from type II Cu-Mo mineralization.

\section{Discussion}

\subsection{Timing of Magmatism and Cu-Mo Mineralization}

Zircon U-Pb and molybdenite Re-Os dating are commonly used to determine the formation age of ore-related intrusion and ore-forming age of hydrothermal deposit $[5,6,8,11,34]$. The zircon LA-ICP-MS U-Pb dating results show that the host rocks of granitic aplite and granite of type I Cu-Mo mineralization were formed at 217.3 $\pm 1.3 \mathrm{Ma}$ and 218.1 $\pm 1.5 \mathrm{Ma}$, respectively (Figure 6b,d). And the host rock of granite of type II mineralization has a similar formation age of $217.1 \pm 1.5 \mathrm{Ma}$ (Figure $6 \mathrm{f}$ ) to the host rocks of type I mineralization. These LA-ICP-MS zircon ages are highly consistent with previous dating results $[2,3,32,49]$. The ore-forming ages of two types of $\mathrm{Cu}$-Mo mineralization have a relatively large age interval. Molybdenite separated from contact surface between granite and granitic aplite (i.e., type I Cu-Mo mineralization) occurred at $217.7 \pm 3.6 \mathrm{Ma}$ (Figure 7b), which is coeval with the emplacement age of granite $(218.1 \pm 1.5 \mathrm{Ma})$ and granitic aplite $(217.3 \pm 1.3 \mathrm{Ma})$. However, the molybdenite of type II Cu-Mo mineralization yield a younger average mean age of $211.8 \pm 4.7 \mathrm{Ma}$ (Figure $7 \mathrm{~d}$ ), which postdates the emplacement event of host granite $(217.1 \pm 1.5 \mathrm{Ma})$. The younger molybdenite Re-Os age of $211.8 \pm 4.7 \mathrm{Ma}$ may indicate that the type II Cu-Mo mineralization was formed in the end stage of magmatic evolution. This is supported by the geological observation that the type II mineralization occurs near granitic pegmatite (Figure $4 b$ ). 


\subsection{Source of Ore-Forming Metals}

Re contents in molybdenite from different hydrothermal deposits vary greatly. Berzina et al. [50] suggested that Re contents of molybdenite may be related to the concentration of Re in ore-forming fluid, the composition of parent magma, physical-chemical conditions (e.g., temperature, pressure, and $f \mathrm{O}_{2}$ ) of crystallization, and sources of ore-forming materials. Based on systematic and comprehensive investigations on different types of endogenous Mo deposit in China, Mao et al. [19] demonstrated that Re content in molybdenite decreases from a mantle source ( $>100 \mathrm{ppm})$, to a mixed mantle/crustal source (10-100 ppm), and to a crustal source ( $<10 \mathrm{ppm}$ ). Similarity, Stein et al. [21] proposed that molybdenite from deposits involved mantle metasomatism, underplating, or melting of mafic/ultramafic rocks generally have high Re contents. In addition, the study showed that deposits originating from intermediate crustal rocks or organic-poor sedimentary rocks are expected to have low Re content [21].

The Re contents in molybdenite from type I Cu-Mo mineralization show large variation ranging from $12.77 \mathrm{ppm}$ to $111.1 \mathrm{ppm}$ (Table 3). Three out of four molybdenites with model ages of 222.3-215.2 Ma have high Re contents, indicating the ore-forming materials were derived from a mantle source. One sample (DC16-67) with youngest model age of 214.8 \pm 3.3 Ma has lowest Re content of $12.77 \mathrm{ppm}$, which probably indicative of crustal contamination of the mineralizing fluid (at $214.8 \pm 3.3 \mathrm{Ma}$ ) after the peak of the magmatic pulse responsible for granite formation and emplacement (at 218.1 $\pm 1.5 \mathrm{Ma}$ ). The type II Cu-Mo mineralization has relatively constant and low Re contents (from 7.983 ppm to $10.40 \mathrm{ppm}$; Table 3), indicative of a mixed mantle and crustal source with a predominantly crustal component.

\subsection{Implication for Regional Exploration}

The EYA is characterized by the occurrence of numerous porphyry and skarn type Cu-Mo-(Au) mineralization in its south segment $[4-6,10-12,14,15,51]$. Based on high-precision zircon $\mathrm{U}-\mathrm{Pb}$ and molybdenite Re-Os geochronology, two episodes of metallogenic events (includes Late Triassic and Late Cretaceous) have been identified in the arc (Figure 8 and Table 4) $[4,6,10,11,14,34]$.

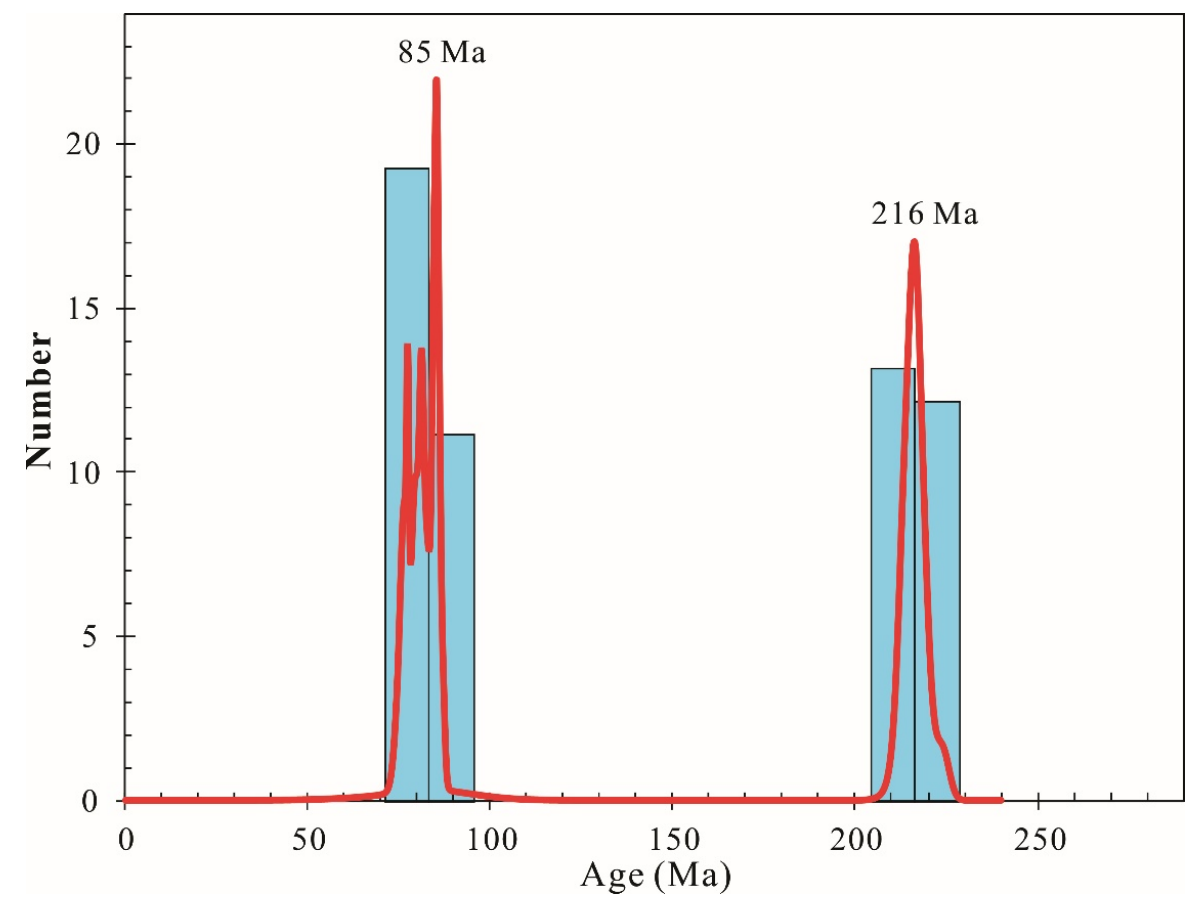

Figure 8. Histogram ages of mineralization of major ore deposits in the EYA. The age data are from Table 4. 
Table 4. Summary of geochronological data for multiple types of metal deposits in the Eastern Yidun arc.

\begin{tabular}{|c|c|c|c|c|c|c|}
\hline \multirow{2}{*}{ Deposit } & \multirow{2}{*}{ Genetic Type } & \multirow{2}{*}{ Metals } & \multicolumn{3}{|c|}{ Age of Mineralization } & \multirow{2}{*}{ Reference } \\
\hline & & & Sample & Method & Age (Ma) & \\
\hline \multicolumn{7}{|c|}{ Northern Eastern Yidun arc } \\
\hline \multirow[t]{3}{*}{ Changdagou } & Porphyry & $\mathrm{Cu}$ & Granodiorite & LA-ICP-MS zircon U-Pb & $216 \pm 0.66 \mathrm{Ma}$ & Zeng Y F. [52] \\
\hline & & $\mathrm{Cu}$ & Granodiorite & LA-ICP-MS zircon U-Pb & $213 \pm 4.4 \mathrm{Ma}$ & Zeng Y F. [52] \\
\hline & & $\mathrm{Cu}$ & Granodiorite & LA-ICP-MS zircon U-Pb & $215 \pm 1 \mathrm{Ma}$ & Li et al. [18] \\
\hline Elong & Porphyry & $\mathrm{Cu}$ & Granodiorite & LA-ICP-MS zircon U-Pb & $208 \pm 4.2 \mathrm{Ma}$ & Zeng Y F. [52] \\
\hline \multicolumn{7}{|c|}{ Southern Eastern Yidun arc } \\
\hline \multirow[t]{13}{*}{ Pulang } & Porphyry & $\mathrm{Cu}-\mathrm{Au}$ & Diorite & SIMS zircon U-Pb & $217.9 \pm 1.8 \mathrm{Ma}$ & Kong et al. [53] \\
\hline & & & & LA-ICP-MS zircon U-Pb & $217.9 \pm 1.8 \mathrm{Ma}$ & Wang et al. [54] \\
\hline & & & Granodiorite & SIMS zircon U-Pb & $216.1 \pm 1.4 \mathrm{Ma}$ & Kong et al. [53] \\
\hline & & & & LA-ICP-MS zircon U-Pb & $214.2 \pm 1.7 \mathrm{Ma}$ & Leng et al. [55] \\
\hline & & & & LA-ICP-MS zircon U-Pb & $216.0 \pm 1.5 \mathrm{Ma}$ & Cao et al. [56] \\
\hline & & & Quartz diorite & SIMS zircon U-Pb & $218.9 \pm 1.3 \mathrm{Ma}$ & Kong et al. [53] \\
\hline & & & & LA-ICP-MS zircon U-Pb & $211.8 \pm 1.9-217.2 \pm 1.4 \mathrm{Ma}$ & Chen et al. [33] \\
\hline & & & & LA-ICP-MS zircon U-Pb & $215.5 \pm 1.6 \mathrm{Ma}$ & Cao et al. [56] \\
\hline & & & & LA-ICP-MS zircon U-Pb & $216.1 \pm 1.7-216.9 \pm 2.0 \mathrm{Ma}$ & Leng et al. [55] \\
\hline & & & Quartz monzonite & SIMS zircon U-Pb & $215.0 \pm 1.3 \mathrm{Ma}$ & Kong et al. [53] \\
\hline & & & & LA-ICP-MS zircon U-Pb & $215.1 \pm 1.3-217.1 \pm 1.8 \mathrm{Ma}$ & Leng et al. [55] \\
\hline & & & & LA-ICP-MS zircon U-Pb & $215.8 \pm 1.3 \mathrm{Ma}$ & Cao et al. [56] \\
\hline & & & Molybdenite & Re-Os isochron & $213 \pm 3.8 \mathrm{Ma}$ & Zeng et al. [13] \\
\hline Lannitang & Porphyry & $\mathrm{Cu}-\mathrm{Au}$ & Quartz monzonite & LA-ICP-MS zircon U-Pb & $216.7 \pm 1.2 \mathrm{Ma}$ & Chen et al. [33] \\
\hline \multirow[t]{2}{*}{ Chundu } & Porphyry & $\mathrm{Cu}$ & Quartz monzonite & LA-ICP-MS zircon U-Pb & $213.1 \pm 1.5-215.3 \pm 2.7 \mathrm{Ma}$ & Chen et al. [33] \\
\hline & & & & LA-ICP-MS zircon U-Pb & $217 \pm 2-218 \pm 2 \mathrm{Ma}$ & Yang et al. [57] \\
\hline \multirow[t]{5}{*}{ Xuejiping } & Porphyry & $\mathrm{Cu}-\mathrm{Mo}$ & Monzonite porphyry & SIMS zircon U-Pb & $218.3 \pm 1.6 \mathrm{Ma}$ & Leng et al. [6] \\
\hline & & & & LA-ICP-MS zircon U-Pb & $213.4 \pm 1.5 \mathrm{Ma}$ & Ren et al. [58] \\
\hline & & & Dioritic porphyry & LA-ICP-MS zircon U-Pb & $214.7 \pm 2.5-216.9 \pm 1.4 \mathrm{Ma}$ & Peng et al. [9] \\
\hline & & & & SHRIMP zircon U-Pb & $215.2 \pm 1.9 \mathrm{Ma}$ & Cao et al. [59] \\
\hline & & & Quartz monzonite & LA-ICP-MS zircon U-Pb & $215.9 \pm 1.4 \mathrm{Ma}$ & Chen et al. [33] \\
\hline \multirow[t]{2}{*}{ Xuejiping } & Porphyry & $\mathrm{Cu}-\mathrm{Mo}$ & Quartz monzonite & LA-ICP-MS zircon U-Pb & $213.4 \pm 1.5 \mathrm{Ma}$ & Cao et al. [56] \\
\hline & & & Molybdenite & Re-Os isochron & $221.4 \pm 2.3 \mathrm{Ma}$ & Leng et al. [6] \\
\hline \multirow{7}{*}{ Hongshan } & Porphyry-skarn & $\mathrm{Cu}-\mathrm{Mo}$ & Granite & LA-ICP-MS zircon U-Pb & $75.0 \pm 1.3 \mathrm{Ma}$ & Cao et al. [56] \\
\hline & & & Quartz monzonite porphyry & LA-ICP-MS zircon U-Pb & $77.3 \pm 0.3 \mathrm{Ma}$ & Peng et al. [9] \\
\hline & & & Granite porphyry & LA-ICP-MS zircon U-Pb & $81.1 \pm 0.5 \mathrm{Ma}$ & Wang et al. [60] \\
\hline & & & & LA-ICP-MS zircon U-Pb & $75.8 \pm 1.3 \mathrm{Ma}$ & Huang et al. [61] \\
\hline & & & Molybdenite & Re-Os isochron & $78.1 \pm 1.9 \mathrm{Ma}$ & Peng et al. [9] \\
\hline & & & Molybdenite & Re-Os isochron & $79.7 \pm 3.1 \mathrm{Ma}$ & Zu et al. [11] \\
\hline & & & Molybdenite & Re-Os model age & $81.9 \pm 1.1 \mathrm{Ma}$ & $\mathrm{Zu}$ et al. [11] \\
\hline
\end{tabular}


Table 4. Cont.

\begin{tabular}{|c|c|c|c|c|c|c|}
\hline \multirow{2}{*}{ Deposit } & \multirow{2}{*}{ Genetic Type } & \multirow{2}{*}{ Metals } & \multicolumn{3}{|c|}{ Age of Mineralization } & \multirow{2}{*}{ Reference } \\
\hline & & & Sample & Method & Age (Ma) & \\
\hline \multirow{8}{*}{ Tongchanggou } & \multirow{8}{*}{ Porphyry-skarn } & \multirow{8}{*}{$\mathrm{Mo}-\mathrm{Cu}$} & Pyrrhotite & Re-Os isochron & $79 \pm 16 \mathrm{Ma}$ & Zu et al. [11] \\
\hline & & & Molybdenite & Re-Os isochron & $77 \pm 2 \mathrm{Ma}$ & Xu et al. [62] \\
\hline & & & Molybdenite & Re-Os isochron & $80.2 \pm 1.3 \mathrm{Ma}$ & Li et al. [7] \\
\hline & & & Molybdenite & Re-Os isochron & $80.0 \pm 1.8 \mathrm{Ma}$ & Meng et al. [63] \\
\hline & & & Molybdenite & Re-Os isochron & $81.2 \pm 2.6 \mathrm{Ma}$ & Wang et al. [14] \\
\hline & & & Granodiorite & LA-ICP-MS zircon U-Pb & $84.3 \pm 0.4-85.2 \pm 0.4 \mathrm{Ma}$ & Yang et al. [51] \\
\hline & & & Molybdenite & Re-Os isochron & $85 \pm 10 \mathrm{Ma}$ & Li et al. [8] \\
\hline & & & Molybdenite & Re-Os model age & $85.2 \pm 0.4-86.8 \pm 0.6 \mathrm{Ma}$ & Yang et al. [51] \\
\hline \multirow[t]{7}{*}{ Xiuwacu } & \multirow[t]{5}{*}{ Hydrothermal quartz-vein } & \multirow[t]{3}{*}{ W-Mo } & Biotite granitic porphyry & LA-ICP-MS zircon U-Pb & $85.6 \pm 0.5 \mathrm{Ma}$ & Wang et al. [14] \\
\hline & & & Monzogranite & LA-ICP-MS zircon U-Pb & $84.8 \pm 0.6 \mathrm{Ma}$ & Wang et al. [14] \\
\hline & & & & LA-ICP-MS zircon U-Pb & $80.2 \pm 1.1 \mathrm{Ma}$ & Cao et al. [56] \\
\hline & & & Alkali-feldspar leucogranite & LA-ICP-MS zircon U-Pb & $84.4 \pm 1.4 \mathrm{Ma}$ & Wang et al. [14] \\
\hline & & & monzogranite porphyry & LA-ICP-MS zircon U-Pb & $86.0 \pm 0.4 \mathrm{Ma}$ & Yang et al. [10] \\
\hline & & & Molybdenite & Re-Os isochron & $83 \pm 1 \mathrm{Ma}$ & Li et al. [34] \\
\hline & & & Molybdenite & Re-Os isochron & $84.7 \pm 0.6 \mathrm{Ma}$ & Yang et al. [10] \\
\hline \multirow[t]{3}{*}{ Relin } & Hydrothermal quartz-vein & $\mathrm{Cu}-\mathrm{Mo}$ & Biotite monzogranite & LA-ICP-MS zircon U-Pb & $76.8 \pm 0.8 \mathrm{Ma}$ & Cao et al. [56] \\
\hline & & & Molybdenite & Re-Os model age & $81.3 \pm 1-84.3 \pm 1.1 \mathrm{Ma}$ & Gao et al. [5] \\
\hline & & & Molybdenite & Re-Os isochron & $81.2 \pm 2.3 \mathrm{Ma}$ & Li et al. [34] \\
\hline
\end{tabular}


The Late Triassic (221-213 Ma) mineralization (e.g., Pulang porphyry Cu-Mo-Au deposit) are genetically associated with Late Triassic subduction-related intermediate-felsic porphyritic intrusions $[4,6,13,24]$. The Late Cretaceous $(88-80 \mathrm{Ma})$ mineralization, including the large Hongshan and Tongchang $\mathrm{Cu}-\mathrm{Mo}$ deposits, have close temporal and spatial relationships with Late Cretaceous I-type granitoids $[4,9,11,14,15]$. The formation of the Late Triassic and Late Cretaceous $\mathrm{Cu}-\mathrm{Mo}-(\mathrm{Au})$ mineralization has been attributed to the westward subduction of Ganze-Litang Paleo-Tethys ocean slab in Late Triassic, and crustal extension in Late Cretaceous, respectively $[4,6,10,12,15]$.

In this study, the type I and tye II Cu-Mo mineralization were dated at $217.7 \pm 3.6 \mathrm{Ma}$ and $211.8 \pm 4.7 \mathrm{Ma}$, respectively, which coincides well with the metallogenic ages of Late Triassic $\mathrm{Cu}-\mathrm{Mo}-(\mathrm{Au})$ mineralization in the SEYA (Table 4). Therefore, we consider that the Cu-Mo mineralization in the NEYA is likely related to the subduction of Ganze-Litang Paleo-Tethys ocean slab in Late Triassic. The subduction-related granitoid in the NEYA is widely exposed, with the outcrops more than $5200 \mathrm{~km}^{2}$ [3]. Previous studies revealed that these rocks belong to high-K calc-alkaline I-type granitoid, which is favorable to the formation of $\mathrm{Cu}-\mathrm{Mo}$ deposits $[64,65]$. Except for the two types $\mathrm{Cu}-\mathrm{Mo}$ mineralization presented in this study, some porphyry $\mathrm{Cu}$ deposits (e.g., Changdagou, Zhujiding; Figure 1a) have also been documented in the NEYA $[16-18,66]$. Previous studies show that the ore-bearing porphyries of Changdagou porphyry $\mathrm{Cu}$ deposit were formed at 216-208 Ma [18,52], which were synchronously formed to the ore-related intrusions in the SEYA. In addition, the Changdagou and Zhujiding porphyry $\mathrm{Cu}$ deposit develop similar hydrothermal alteration (e.g., silification, propylitization, phyllic and potassic alteration), and ore fabric features (e.g., veinlet and disseminated ore structures) to those of porphyry $\mathrm{Cu}-\mathrm{Mo}$ deposits in the SEYA $[16-18,52,66]$. Recognition of these porphyry $\mathrm{Cu}$ deposits and $\mathrm{Cu}-\mathrm{Mo}$ mineralization indicate that the NEYA exists Late Triassic Cu-Mo metallogenesis. Therefore, a renewed exploration should be encouraged to find late Triassic Cu-Mo resources in the NEYA.

\section{Conclusions}

Molybdenite Re-Os dating indicates that the type I and type II Cu-Mo mineralization occurred at $\sim 218 \mathrm{Ma}$ and $\sim 212 \mathrm{Ma}$, respectively. Zircon LA-ICP-MS U-Pb dating shows that the granite and granitic aplite associated with type I Cu-Mo mineralization were formed at $218.1 \pm 1.5 \mathrm{Ma}$ and $217.3 \pm 1.3 \mathrm{Ma}$, respectively. The host granite of type II Cu-Mo mineralization was formed at 217.1 $\pm 1.5 \mathrm{Ma}$. Re content in molybdenite suggests that the ore-forming materials of type I Cu-Mo mineralization were derived from a mantle source, while the type II Cu-Mo mineralization was sourced from a mixed mantle and crustal source. The relatively low Re contents and younger Re-Os ages of molybdenite in type II mineralization may indicate that type II Cu-Mo mineralization was formed in the late stage of magmatic evolution, accompanying with addition of crustal-derived materials. This study provides significant evidence to support that the NEYA hosts Late Triassic Cu-Mo mineralization. Recognition of Late Triassic porphyry $\mathrm{Cu}$ deposits and $\mathrm{Cu}-\mathrm{Mo}$ mineralization in the NEYA should encourage renewed investigations and ore prospecting in the NEYA to find late Triassic Cu-Mo deposits.

Author Contributions: Z.-D.T. and C.-B.L. wrote the manuscript. C.-B.L. designed this project, and X.-C.Z. helped interpret the data. L.-M.Z. and Y.-W.T. helped molybdenite Re-Os and zircon U-Pb dating, respectively.

Funding: This work was supported by the National Key R\&D Program of China (2016YFC0600305), and NSFC projects $(41673051,41373051)$.

Acknowledgments: The authors are grateful to the four anonymous reviewers for their constructive comments, which greatly improved the manuscript.

Conflicts of Interest: The authors declare no conflict of interest. 


\section{References}

1. Leng, C.B.; Huang, Q.Y.; Zhang, X.C.; Wang, S.X.; Zhong, H.; Hu, R.Z.; Bi, X.W.; Zhu, J.J.; Wang, X.S. Petrogenesis of the Late Triassic volcanic rocks in the Southern Yidun arc, SW China: Constraints from the geochronology, geochemistry, and Sr-Nd-Pb-Hf isotopes. Lithos 2014, 190, 363-382. [CrossRef]

2. Reid, A.; Wilson, C.J.L.; Shun, L.; Pearson, N.; Belousova, E. Mesozoic plutons of the Yidun Arc, SW China: $\mathrm{U} / \mathrm{Pb}$ geochronology and Hf isotopic signature. Ore Geol. Rev. 2007, 31, 88-106. [CrossRef]

3. He, D.F.; Zhu, W.G.; Zhong, H.; Ren, T.; Bai, Z.J.; Fan, H.P. Zircon U-Pb geochronology and elemental and Sr-Nd-Hf isotopic geochemistry of the Daocheng granitic pluton from the Yidun Arc, SW China. J. Asian Earth Sci. 2013, 67, 1-17. [CrossRef]

4. Li, W.C.; Yu, H.J.; Gao, X.; Liu, X.L.; Wang, J.H. Review of Mesozoic multiple magmatism and porphyry Cu-Mo (W) mineralization in the Yidun Arc, eastern Tibet Plateau. Ore Geol. Rev. 2017, 90, 795-812. [CrossRef]

5. Gao, X.; Yang, L.Q.; Meng, J.Y.; Zhang, L.J. Zircon U-Pb, molybdenite Re-Os geochronology and $\mathrm{Sr}-\mathrm{Nd}-\mathrm{Pb}-\mathrm{Hf}-\mathrm{O}-\mathrm{S}$ isotopic constraints on the genesis of Relin Cu-Mo deposit in Zhongdian, Northwest Yunnan, China. Ore Geol. Rev. 2017, 91, 945-962. [CrossRef]

6. Leng, C.B.; Zhang, X.C.; Hu, R.Z.; Wang, S.X.; Zhong, H.; Wang, W.Q.; Bi, X.W. Zircon U-Pb and molybdenite Re-Os geochronology and $\mathrm{Sr}-\mathrm{Nd}-\mathrm{Pb}-\mathrm{Hf}$ isotopic constraints on the genesis of the Xuejiping porphyry copper deposit in Zhongdian, Northwest Yunnan, China. J. Asian Earth Sci. 2012, 60, 31-48. [CrossRef]

7. Li, W.C.; Yin, G.H.; Yu, H.J.; Lu, Y.X.; Liu, X.L. The porphyry metallogenesis of Geza volcanic magmatic arc in NW Yunnan. Acta Petrol. Sin. 2011, 27, 2541-2552, (In Chinese with English abstract).

8. Li, W.C.; Yu, H.J.; Yin, G.H.; Cao, X.M.; Huang, D.Z.; Dong, T. Re-Os dating of molybdenite from Tongchanggou Mo-Polymetallic deposit in northwest Yunnan and its metallogenic environment. Miner. Depos. 2012, 31, 282-292, (In Chinese with English abstract).

9. Peng, H.J.; Mao, J.W.; Pei, R.F.; Zhang, C.Q.; Tian, G.; Zhou, Y.M.; Li, J.X.; Hou, L. Geochronology of the Hongniu-Hongshan porphyry and skarn $\mathrm{Cu}$ deposit, northwestern Yunnan province, China: Implications for mineralization of the Zhongdian arc. J. Asian Earth Sci. 2014, 79, 682-695. [CrossRef]

10. Yang, L.Q.; Gao, X.; Shu, Q.H. Multiple Mesozoic porphyry-Skarn Cu (Mo-W) systems in Yidun Terrane, east Tethys: Constraints from zircon U-Pb and molybdenite Re-Os geochronology. Ore Geol. Rev. 2017, 90, 813-826. [CrossRef]

11. Zu, B.; Xue, C.J.; Zhao, Y.; Qu, W.J.; Li, C.; Symons, D.T.A.; Du, A.D. Late Cretaceous metallogeny in the Zhongdian area: Constraints from Re-Os dating of molybdenite and pyrrhotite from the Hongshan $\mathrm{Cu}$ deposit, Yunnan, China. Ore Geol. Rev. 2015, 64, 1-12. [CrossRef]

12. Li, W.C.; Zeng, P.S.; Hou, Z.Q.; White, N.C. The Pulang Porphyry Copper Deposit and Associated Felsic Intrusions in Yunnan Province, Southwest China. Econ. Geol. 2011, 106, 79-92.

13. Zeng, P.S.; Li, W.C.; Wang, H.P.; Li, H. The Indosinian Pulang superlarge porphyry copper deposit in Yunnan, China: Petrology and chronology. Acta Petrol. Sin. 2006, 22, 989-1000, (In Chinese with English abstract).

14. Wang, X.S.; Bi, X.W.; Leng, C.B.; Zhong, H.; Tang, H.F.; Chen, Y.W.; Yin, G.H.; Huang, D.Z.; Zhou, M.F. Geochronology and geochemistry of Late Cretaceous igneous intrusions and Mo-Cu-(W) mineralization in the southern Yidun Arc, SW China: Implications for metallogenesis and geodynamic setting. Ore Geol. Rev. 2014, 61, 73-95. [CrossRef]

15. Wang, X.S.; Hu, R.Z.; Bi, X.W.; Leng, C.B.; Pan, L.C.; Zhu, J.J.; Chen, Y.W. Petrogenesis of Late Cretaceous I-Type granites in the southern Yidun Terrane: New constraints on the Late Mesozoic tectonic evolution of the eastern Tibetan Plateau. Lithos 2014, 208, 202-219. [CrossRef]

16. Chen, X.; Li, Y.G.; Xu, M.; Reng, G.M. Geology and ore genesis of Zhujiding porphyry copper in western. Sichuan Province. Geol. Prospect. 2008, 44, 42-45, (In Chinese with English abstract).

17. Deng, H.B. Geological features of the Zhujiding porphyry copper deposit in Daocheng, Sichuan. Acta Geol. Sichuan. 2008, 28, 105-108, (In Chinese with English abstract).

18. Li, X.Y.; Zhang, J.R.; Song, H.; Lai, C.K. Copper mineralization potential of Late Triassic granitoids in Northern Yidun Arc, SW China. Minerals 2019, 9, 337. [CrossRef]

19. Mao, J.W.; Zhang, Z.C.; Zhang, Z.H.; Du, A.D. Re-Os isotopic dating of molybdenites in the Xiaoliugou W (Mo) deposit in the northern Qilian mountains and its geological significance. Geochim. Cosmochim. Acta 1999, 63, 1815-1818. 
20. Stein, H.J.; Markey, R.J.; Morgan, J.W.; Du, A.D.; Sun, Y.L. Highly precise and accurate Re-Os ages for molybdenite from the East Qinling molybdenum belt, Shanxi Province, China. Econ. Geol. 1997, 92, 827-835. [CrossRef]

21. Stein, H.J.; Markey, R.J.; Morgan, J.W.; Hannah, J.L.; Schersten, A. The remarkable Re-Os chronometer in molybdenite: How and why it works. Terra Nova 2001, 13, 479-486. [CrossRef]

22. Reid, A.J.; Wilson, C.J.L.; Liu, S. Structural evidence for the Permo-Triassic tectonic evolution of the Yidun Arc, eastern Tibetan Plateau. J. Struct. Geol. 2005, 27, 119-137. [CrossRef]

23. BGMRSP (Bureau of Geology and Mineral Resources of Sichuan Province). Regional Geology of the Sichuan Province; Geological Publishing House: Beijing, China, 1991; pp. 1-730, (In Chinese with English abstract).

24. Hou, Z.Q.; Hou, L.W.; Ye, Q.T.; Liu, F.L.; Tang, G.G. Tectono-Magmatic Evolution and Volcanogenic Massive Sulphide Deposits in the Yidun Island-Arc, Sanjiang Region, China; Earthquake Publishing House: Beijing, China, 1995; pp. 1-218, (In Chinese with English abstract).

25. Chang, E.Z. Geology and tectonics of the Songpan-Ganzi fold belt, southwestern China. Int. Geol. Rev. 2000, 42, 813-831. [CrossRef]

26. Song, X.Y.; Zhou, M.F.; Cao, Z.M.; Robinson, P.T. Late Permian rifting of the South China Craton caused by the Emeishan mantle plume. J. Geol. Soc. 2004, 161, 773-781. [CrossRef]

27. Tian, Z.D.; Leng, C.B.; Zhang, X.C.; Yin, C.J.; Zhang, W.; Guo, J.H.; Tian, F. Mineralogical characteristics of chlorites from Precambrian metamorphic rocks in Yidun magmatic arc of Qinghai-Tibet Plateau and their geological implications. J. Earth Sci. Env. 2018, 40, 36-48, (In Chinese with English abstract).

28. Tian, Z.D.; Leng, C.B.; Zhang, X.C.; Yin, C.J.; Zhang, W.; Guo, J.H.; Chen, L.H. Mineralogical and petrogeochemical characteristics of the metamorphic basement of Yidun Terrane and their geological implications. Acta Mineral. Sin. 2018, 38, 152-165, (In Chinese with English abstract).

29. Wang, B.Q.; Wang, W.; Zhou, M.F. Provenance and tectonic setting of the Triassic Yidun Group, the Yidun Terrane, Tibet. Geosci. Front. 2013, 4, 765-777. [CrossRef]

30. Wang, B.Q.; Zhou, M.F.; Chen, W.T.; Gao, J.F.; Yan, D.P. Petrogenesis and tectonic implications of the Triassic volcanic rocks in the northern Yidun Terrane, Eastern Tibet. Lithos 2013, 175, 285-301. [CrossRef]

31. Hou, Z.Q.; Qu, X.M.; Zhou, J.R.; Yang, Y.Q.; Huang, D.H.; Lv, Q.T.; Tang, S.H.; Yu, J.J.; Wang, H.P.; Zhao, J.H. Collision-Orogenic processes of the Yidun arc in the Sanjiang region: Record of granites. Acta Geol. Sin. 2001, 75, 484-497, (In Chinese with English abstract).

32. Wu, T.; Xiao, L.; Wilde, S.A.; Ma, C.Q.; Zhou, J.X. A mixed source for the Late Triassic Garze-Daocheng granitic belt and its implications for the tectonic evolution of the Yidun arc belt, eastern Tibetan Plateau. Lithos 2017, 288, 214-230. [CrossRef]

33. Chen, J.L.; Xu, J.F.; Ren, J.B.; Huang, X.X.; Wang, B.D. Geochronology and geochemical characteristics of Late Triassic porphyritic rocks from the Zhongdian arc, eastern Tibet, and their tectonic and metallogenic implications. Gondwana Res. 2014, 26, 492-504. [CrossRef]

34. Li, J.K.; Li, W.C.; Wang, D.H.; Lu, Y.X.; Yin, G.H.; Xue, S.R. Re-Os dating for ore-Forming event in the late of Yanshan epoch and research of ore-Forming regularity in Zhongdian Arc. Acta Petrol. Sin. 2007, 23, 2415-2422, (In Chinese with English abstract).

35. Chen, W.S.; Zheng, S.Q.; Li, S.L.; Wen, P.R.; Zheng, Y.M. 1:200,000 Geological Map of Daocheng, Sichuan Province; Bureau of Geology, Sichuan: Chengdu, China, 1984.

36. Gao, Y.T.; Jia, Z.Q.; Li, X.Z.; Wen, P.R.; Mao, J.Y. 1:200,000 Geological Map of Ganze, Sichuan Province; Bureau of Geology, Sichuan: Chengdu, China, 1980.

37. Wiedenbeck, M.; Alle, P.; Corfu, F.; Griffin, W.; Meier, M.; Oberli, F.; Quadt, A.; Roddick, J.C.; Spiegel, W. Three natural zircon standards for U-Th-Pb, Lu-Hf, trace element and REE analyses. Geostand. Newsl. 1995, 19, 1-23. [CrossRef]

38. Jackson, S.E.; Pearson, N.J.; Griffin, W.L.; Belousova, E.A. The application of laser ablation-Inductively coupled plasma-Mass spectrometry to in situ U-Pb zircon geochronology. Chem. Geol. 2004, 211, 47-69. [CrossRef]

39. Slama, J.; Kosler, J.; Condon, D.J.; Crowley, J.L.; Gerdes, A.; Hanchar, J.M.; Horstwood, M.S.A.; Morris, G.A.; Nasdala, L.; Norberg, N.; et al. Plesovice zircon-A new natural reference material for $\mathrm{U}-\mathrm{Pb}$ and Hf isotopic microanalysis. Chem. Geol. 2008, 249, 1-35. [CrossRef] 
40. Liu, Y.S.; Gao, S.; Hu, Z.C.; Gao, C.G.; Zong, K.Q.; Wang, D.B. Continental and oceanic crust recycling-Induced melt-Peridotite interactions in the Trans-North China Orogen: U-Pb dating, Hf isotopes and trace elements in zircons from mantle xenoliths. J. Petrol. 2010, 51, 537-571. [CrossRef]

41. Liu, Y.S.; Hu, Z.C.; Gao, S.; Gunther, D.; Xu, J.; Gao, C.G.; Chen, H.H. In situ analysis of major and trace elements of anhydrous minerals by LA-ICP-MS without applying an internal standard. Chem. Geol. 2008, 257, 34-43. [CrossRef]

42. Ludwig, K.R. User's Manual for Isoplot 3.00, a Geochronological Toolkit for Microsoft Excel; Berkeley Geochronology Center Special Publication: Berkeley, CA, USA, 2003; pp. 25-32.

43. Du, A.D.; Wu, S.Q.; Sun, D.Z.; Wang, S.X.; Qu, W.J.; Markey, R.; Stain, H.; Morgan, J.; Malinovskiy, D. Preparation and certification of Re-Os dating reference materials: Molybdenites HLP and JDC. Geostand. Geoanal. Res. 2004, 28, 41-52. [CrossRef]

44. Shirey, S.B.; Walker, R.J. Carius tube digestion for low-Blank rhenium-Osmium analysis. Anal. Chem. 1995, 67, 2136-2141. [CrossRef]

45. Smoliar, M.I.; Walker, R.J.; Morgan, J.W. Re-Os ages of group IIA, IIIA, IVA, and IVB iron meteorites. Science 1996, 271, 1099-1102. [CrossRef]

46. Corfu, F.; Hanchar, J.M.; Hoskin, P.W.O.; Kinny, P. Atlas of zircon textures. Rev. Mineral. Geochem. 2003, 53, 469-500. [CrossRef]

47. Hanchar, J.M.; Rudnick, R.L. Revealing hidden structures: The application of cathodoluminescence and back-scattered electron imaging to dating zircons from lower crustal xenoliths. Lithos 1995, 36, 289-303. [CrossRef]

48. Wu, Y.B.; Zheng, Y.F. Genesis of zircon and its constraints on interpretation of U-Pb age. Chin. Sci. Bull. 2004, 49, 1554-1569. [CrossRef]

49. Weislogel, A.L. Tectonostratigraphic and geochronologic constraints on evolution of the northeast Paleotethys from the Songpan-Ganzi complex, central China. Tectonophysics 2008, 451, 331-345. [CrossRef]

50. Berzina, A.N.; Sotnikov, V.I.; Economou-Eliopoulos, M.; Eliopoulos, D.G. Distribution of rhenium in molybdenite from porphyry $\mathrm{Cu}-\mathrm{Mo}$ and Mo-Cu deposits of Russia (Siberia) and Mongolia. Ore Geol. Rev. 2005, 26, 91-113. [CrossRef]

51. Yang, L.Q.; Deng, J.; Gao, X.; He, W.Y.; Meng, J.Y.; Santosh, M.; Yu, H.J.; Yang, Z.; Wang, D. Timing of formation and origin of the Tongchanggou porphyry-Skarn deposit: Implications for Late Cretaceous Mo-Cu metallogenesis in the southern Yidun Terrane, SE Tibetan Plateau. Ore Geol. Rev. 2017, 81, 1015-1032. [CrossRef]

52. Zeng, Y.F. The Geological Characteristics and Discussions on the Geneis of Porphyry Copper Deposit in Changdagou. Master's Thesis, Chengdu University of Technology, Chengdu, China, 2015.

53. Kong, D.X.; Xu, J.F.; Chen, J.L. Oxygen isotope and trace element geochemistry of zircons from porphyry copper system: Implications for Late Triassic metallogenesis within the Yidun Terrane, southeastern Tibetan Plateau. Chem. Geol. 2016, 441, 148-161. [CrossRef]

54. Wang, B.Q.; Zhou, M.F.; Li, J.W.; Yan, D.P. Late Triassic porphyritic intrusions and associated volcanic rocks from the Shangri-La region, Yidun terrane, Eastern Tibetan Plateau: Adakitic magmatism and porphyry copper mineralization. Lithos 2011, 127, 24-38. [CrossRef]

55. Leng, C.B.; Gao, J.F.; Chen, W.T.; Zhang, X.C.; Tian, Z.D.; Guo, J.H. Platinum-Group elements, zircon Hf-O isotopes, and mineralogical constraints on magmatic evolution of the Pulang porphyry Cu-Au system, SW China. Gondwana Res. 2018, 62, 163-177. [CrossRef]

56. Cao, K.; Xu, J.F.; Chen, J.L.; Huang, X.X.; Ren, J.B.; Zhao, X.D.; Liu, Z.X. Double-Layer structure of the crust beneath the Zhongdian arc, SW China: U-Pb geochronology and Hf isotope evidence. J. Asian Earth Sci. 2016, 115, 455-467. [CrossRef]

57. Yang, F.; Zou, G.F.; Wu, J.; Li, F.; Jiang, Y.G.; Zhao, X.D. Ages and geological significance of the porphyries in the Chundu copper mining area in Zhongdian, Yunnan Province. Geotecton. Metallog. 2011, 35, 314-317, (In Chinese with English abstract).

58. Ren, J.B.; Xu, J.F.; Chen, J.L. Zircon geochronology and geological implications of ore-Bearing porphyries fromZhongdian arc. Acta Petrol. Sin. 2011, 27, 2591-2599, (In Chinese with English abstract).

59. Cao, D.H.; Wang, A.J.; Huang, Y.F.; Zhang, W.; Hou, K.J.; Li, R.P.; Li, Y.K. SHRIMP geochronology and Hf isotope composition of zircons from Xuejiping porphyry copper deposit, Yunnan Province. Acta Geol. Sin. 2009, 83, 1430-1435, (In Chinese with English abstract). 
60. Wang, X.S.; Bi, X.W.; Leng, C.B.; Tang, Y.Y.; Lan, J.B.; Qi, Y.Q.; Shen, N.P. LA-ICP-MS zircon U-Pb dating of granite porphyry in the Hongshan Cu-Polymetallic deposit, Zhongdian, northwest Yunnan, China and its geological implication. Acta Mineral. Sin. 2011, 31, 315-321, (In Chinese with English abstract).

61. Huang, X.X.; Xu, J.F.; Chen, J.L.; Ren, J.B. Geochronology, geochemistry, and petrogenesis of two periods of intermediate-Acid intrusive rocks from Hongshan area in Zhongdian arc. Acta Petrol. Sin. 2012, 28, 1493-1506, (In Chinese with English abstract).

62. Xu, X.W.; Cai, X.P.; Qu, W.J.; Song, B.C.; Qin, K.Z.; Zhang, B.L. Late Cretaceous granite porphyritic Cu-Mo mineralization system in the Hongshan area, Northwestern Yunnan and its significances for tectonics. Acta Geol. Sin. 2006, 80, 1422-1433, (In Chinese with English abstract).

63. Meng, J.Y.; Yang, L.Q.; Lu, L.; Gao, X.; Li, J.X.; Luo, Y.Z. Re-Os dating of molybdenite from the Hongshan Cu-Mo deposit in Northwest Yunnan and its implications for mineralization. Acta Petrol. Sin. 2013, 29, 1214-1222, (In Chinese with English abstract).

64. Meinert, L.D.; Dipple, G.M.; Nicolescu, S. World Skarn Deposits. Econ. Geol. 2005, 1905-2005, $299-336$.

65. Wan, B.; Xiao, W.J.; Han, C.M.; Windley, B.F.; Zhang, L.C.; Qu, W.J.; Du, A.D. Re-Os molybdenite age of the $\mathrm{Cu}-\mathrm{Mo}$ skarn ore deposit at Suoerkuduke in East Junggar, NW China and its geological significance. Ore Geol. Rev. 2014, 56, 541-548. [CrossRef]

66. Fei, G.C.; Li, Y.G. Geological features and mineragenic potentiality of granite aplite in Zhujiding copper deposit in Sichuan Province. Xinjiang Geol. 2010, 28, 204-208, (In Chinese with English abstract).

(C) 2019 by the authors. Licensee MDPI, Basel, Switzerland. This article is an open access article distributed under the terms and conditions of the Creative Commons Attribution (CC BY) license (http://creativecommons.org/licenses/by/4.0/). 